\title{
Merging Dirac points and topological phase transitions in the tight-binding model on the generalized honeycomb lattice
}

\author{
Yasumasa Hasegawa \\ Department of Material Science, Graduate School of Material Science, University of Hyogo, Hyogo, 678-1297, Japan \\ Keita Kishigi \\ Faculty of Education, Kumamoto University, Kurokami 2-40-1, Kumamoto, 860-8555, Japan
}

(Dated: July 30, 2012)

\begin{abstract}
Moving, merging and annihilating Dirac points are studied theoretically in the tight-binding model on honeycomb lattice with up-to third-nearest-neighbor hoppings. We obtain a rich phase diagram of the topological phase transitions in the parameter space of direction-dependent hoppings. We obtain the conditions for the three Dirac points to merge and for the tricritical points. We find that only very small third-nearest-neighbor hoppings are enough for the existence of the merging of three-Dirac-points and the tricritical points, if the system is sufficiently anisotropic. The density of states is obtained to be $D(\epsilon) \propto|\epsilon|^{1 / 3}$ when three Dirac points merge, and $D(\epsilon) \propto|\epsilon|^{1 / 4}$ at the tricritical points. It is possible to realize these topological phase transitions in the ultracold atoms on the optical lattice, strained monolayer graphene or strained bilayer graphene.
\end{abstract}

PACS numbers: 73.22.Pr, 73.43.Nq, 71.10.Pm, 73.20.At

\section{INTRODUCTION}

Massless Dirac fermions are realized in various fields in condensed matter physics and they attract much interest recently, e.g. graphene $\mathrm{e}^{1-3}$, the organic conductor $\alpha$-(BEDT-TTF $)_{2} \mathrm{I}_{3}{ }^{4,5}$ and iron-based superconductor, $\mathrm{BaFe}_{2} \mathrm{As}_{2}{ }^{6}$. These materials have Dirac points and the linear band structure near the Fermi energy.

The moving, merging and annihilating Dirac points due to the breaking of the rotational symmetry were studied $^{7}$ and observed recently in the ultracold atoms on a optical lattice ${ }^{8}$. Many authors have theoretically studied the merging Dirac points in $\alpha$-(BEDT-TTF $)_{2} \mathrm{I}_{3}{ }^{9}$, strained or twisted bilayer graphene $\mathrm{e}^{10-14}$, and the honeycomb lattice with third-nearest-neighbor hoppings ${ }^{15}$. Most of the studies have used the effective model, which describe the energy near the Dirac points.

The energy band of graphene is obtained by firstprinciples band calculations ${ }^{16-18}$, and the band structure near the Fermi energy can be reproduced by a simple tight-binding model ${ }^{19}$. If hoppings are only between the nearest sites and they are independent of the direction, there are two Dirac points at $\mathbf{K}$ and $\mathbf{K}^{\prime}$ in the Brillouin zone. Reich et al. ${ }^{20}$ have shown that up to third-nearestneighbor hoppings are necessary to describe the band structure in all Brillouin zone. They have obtained that the nearest-neighbor, the next-nearest-neighbor and the third-nearest-neighbor hoppings are 2.79-2.97 eV, 0.073$0.68 \mathrm{eV}$ and $0.3-0.33 \mathrm{eV}$, respectively ${ }^{20}$.

A finite gap is important for the application to nano devices. There are two routes to open the finite gap at the Dirac points. One route is via the the violation of the inversion symmetry. The violation of the inversion symmetry can be caused by the difference of the on-site potentials on A and B sublattices or the sublattice-dependent second-neighbor hoppings ${ }^{21,22}$. The finite gap observed experimentally in graphene on a $\mathrm{SiC}$ substrate ${ }^{23}$ is caused by the breaking of the inversion symmetry.

The other route to open a finite gap is realized by making two Dirac points with opposite topological number merge and annihilate $e^{7,9-15}$. When the hoppings between nearest sites become different in three directions, the Dirac points move from $\mathbf{K}$ and $\mathbf{K}^{\prime}$ to one of the three inequivalent $M$ points $\left(\mathbf{M}_{1}, \mathbf{M}_{2}\right.$ and $\left.\mathbf{M}_{3}\right)$, merge into a semi-Dirac point and annihilate to make a finite energy gap. In order to make a finite gap in monolayer graphene, we need the deformations of the order of $20 \%$ caused by the strong uniaxial strain ${ }^{24}$ or the shear $\operatorname{strain}^{25}$, which are difficult to realize. However, the parameters can be controlled in the ultracold atoms on the optical lattice and the merging and annihilating Dirac points are observed $^{8}$.

It has been shown that the strained or twisted bilayer graphene is modeled by the single-layer honeycomb lattice with the third-nearest-neighbor hoppings, the strength of which are near the half of the nearestneighbor hoppings ${ }^{14}$.

In this paper we study the tight-binding model on the honeycomb lattice with up-to third-nearest-neighbor hoppings, and we study the moving and the merging of the Dirac points by changing the strength of directiondependent hoppings. We obtain interesting phase diagrams, in which we can see the merging of three Dirac points and the tricritical points. The density of states due to Dirac points and merged Dirac points are calculated.

The model is given in Section II. The topological number and the Berry phase of the Dirac points are discussed in Section III. In Section IV we study the phase diagram in which we consider only the phase transition due to the merging of two Dirac points at $\mathbf{M}_{1}, \mathbf{M}_{2}, \mathbf{M}_{3}$, and $\boldsymbol{\Gamma}$. Moving Dirac points are studied in the model with direction-dependent nearest-neighbor hoppings in Section V. Rich phase diagrams in the model with direction- 

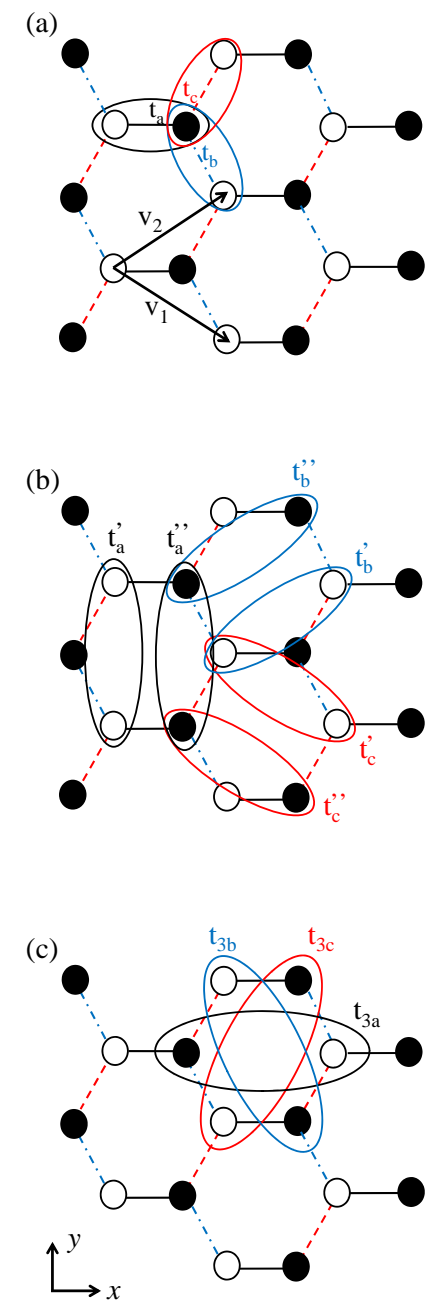

FIG. 1. (Color online) Honeycomb lattice and hoppings. Filled circles $(\bullet)$ and open circles (o) are sites in A sublattice and $\mathrm{B}$ sublattice, respectively. $v_{1}$ and $v_{2}$ are the unit vectors. The hoppings between the nearest sites, the nextnearest sites, and the third-nearest sites are shown in (a), (b), and (c), respectively, and they are considered to depend on the direction and the sublattices.

dependent nearest-neighbor and third-nearest-neighbor hoppings are studied in Section VI. Section VII is the conclusions and detailed calculations are given in Appendix.

\section{TIGHT-BINDING MODEL ON HONEYCOMB LATTICE}

We study the tight-binding electrons on the honeycomb lattice (see Fig. 1). There are two sublattices, A and B (open and filled circles in Fig. 1), which form triangular lattices. We take the unit vectors as

$$
\mathbf{v}_{1}=\left(\frac{\sqrt{3}}{2} a,-\frac{1}{2} a\right)
$$

and

$$
\mathbf{v}_{2}=\left(\frac{\sqrt{3}}{2} a, \frac{1}{2} a\right)
$$

and the vectors connecting the nearest sites as

$$
\begin{aligned}
& \boldsymbol{\delta}_{a}=\frac{1}{3}\left(\mathbf{v}_{1}+\mathbf{v}_{2}\right)=\left(\frac{\sqrt{3}}{3} a, 0\right) \\
& \boldsymbol{\delta}_{b}=\frac{1}{3}\left(\mathbf{v}_{2}-2 \mathbf{v}_{1}\right)=\left(-\frac{\sqrt{3}}{6} a, \frac{1}{2} a\right),
\end{aligned}
$$

and

$$
\boldsymbol{\delta}_{c}=\frac{1}{3}\left(\mathbf{v}_{1}-2 \mathbf{v}_{2}\right)=\left(-\frac{\sqrt{3}}{6} a,-\frac{1}{2} a\right),
$$

where $a$ is the lattice constant. Hereafter, we set $a=1$.

We consider the direction-dependent hoppings between nearest sites $\left(t_{a}, t_{b}\right.$, and $\left.t_{c}\right)$, the next-nearest sites $\left(t_{a}^{\prime}, t_{b}^{\prime}\right.$, $t_{c}^{\prime}, t_{a}^{\prime \prime}, t_{b}^{\prime \prime}$ and $\left.t_{c}^{\prime \prime}\right)$ and the third-nearest sites $\left(t_{3 a}, t_{3 b}\right.$, and $\left.t_{3 c}\right)$, as shown in Fig. 1. The hoppings between the nextnearest sites within the A sublattice are labeled by $t_{a}^{\prime}, t_{b}^{\prime}$ and $t_{c}^{\prime}$, and those within the $\mathrm{B}$ sublattice are labeled by $t_{a}^{\prime \prime}, t_{b}^{\prime \prime}$ and $t_{c}^{\prime \prime}$. The Hamiltonian is given by

$$
\mathcal{H}=\sum_{\mathbf{k}} c_{\mathbf{k}}^{\dagger} \mathcal{E}_{\mathbf{k}} c_{\mathbf{k}}
$$

where

$$
c_{\mathbf{k}}=\left(\begin{array}{c}
a_{\mathbf{k}} \\
b_{\mathbf{k}}
\end{array}\right)
$$

$a_{\mathbf{k}}$ and $b_{\mathbf{k}}$ are the annihilation operators at $A$ and $B$ sublattices, respectively,

$$
\mathcal{E}_{\mathbf{k}}=\sum_{\mu=0,1,2,3} \epsilon_{\mu}(\mathbf{k}) \sigma_{\mu}
$$

$\sigma_{0}$ is the $2 \times 2$ unit matrix, $\sigma_{j}(j=1,2$, and 3$)$ are Pauli matrices and

$$
\begin{aligned}
\epsilon_{0}(\mathbf{k})= & \frac{1}{2}\left(\epsilon_{A}+\epsilon_{B}\right)-\left(t_{c}^{\prime}+t_{c}^{\prime \prime}\right) \cos \left(\mathbf{v}_{1} \cdot \mathbf{k}\right) \\
& -\left(t_{b}^{\prime}+t_{b}^{\prime \prime}\right) \cos \left(\mathbf{v}_{2} \cdot \mathbf{k}\right) \\
& -\left(t_{a}^{\prime}+t_{a}^{\prime \prime}\right) \cos \left(\left(\mathbf{v}_{2}-\mathbf{v}_{1}\right) \cdot \mathbf{k}\right) \\
\epsilon_{1}(\mathbf{k})= & -t_{a} \cos \left(\boldsymbol{\delta}_{a} \cdot \mathbf{k}\right)-t_{b} \cos \left(\boldsymbol{\delta}_{b} \cdot \mathbf{k}\right) \\
& -t_{c} \cos \left(\boldsymbol{\delta}_{c} \cdot \mathbf{k}\right) \\
& -t_{3 a} \cos \left(2 \boldsymbol{\delta}_{a} \cdot \mathbf{k}\right)-t_{3 b} \cos \left(2 \boldsymbol{\delta}_{b} \cdot \mathbf{k}\right) \\
& -t_{3 c} \cos \left(2 \boldsymbol{\delta}_{c} \cdot \mathbf{k}\right) \\
\epsilon_{2}(\mathbf{k})= & -t_{a} \sin \left(\boldsymbol{\delta}_{a} \cdot \mathbf{k}\right)-t_{b} \sin \left(\boldsymbol{\delta}_{b} \cdot \mathbf{k}\right) \\
& -t_{c} \sin \left(\boldsymbol{\delta}_{c} \cdot \mathbf{k}\right) \\
& -t_{3 a} \sin \left(-2 \boldsymbol{\delta}_{a} \cdot \mathbf{k}\right)-t_{3 b} \sin \left(-2 \boldsymbol{\delta}_{b} \cdot \mathbf{k}\right) \\
& -t_{3 c} \sin \left(-2 \boldsymbol{\delta}_{c} \cdot \mathbf{k}\right), \\
1 & \left(\epsilon_{A}-\epsilon_{B}\right)-\left(t_{c}^{\prime}-t_{c}^{\prime \prime}\right) \cos \left(\mathbf{v}_{1} \cdot \mathbf{k}\right) \\
\epsilon_{3}(\mathbf{k})= & \left(t_{b}^{\prime}-t_{b}^{\prime \prime}\right) \cos \left(\mathbf{v}_{2} \cdot \mathbf{k}\right) \\
& -\left(t_{a}^{\prime}-t_{a}^{\prime \prime}\right) \cos \left(\left(\mathbf{v}_{2}-\mathbf{v}_{1}\right) \cdot \mathbf{k}\right)
\end{aligned}
$$


The energy is given by

$$
E_{ \pm}(\mathbf{k})=\epsilon_{0}(\mathbf{k}) \pm \sqrt{\sum_{j=1}^{3}\left(\epsilon_{j}(\mathbf{k})\right)^{2}} .
$$

The nearest-neighbor hoppings and the third-nearestneighbor hoppings connect the sites in A and B sublattices, while the next-nearest hoppings connect sites within A sublattice or B sublattice. Therefore, the nearest hoppings and the third-nearest-neighbor hoppings appear only in the off-diagonal elements (Eqs. (10) and (11)), and the next-nearest hoppings appear only in the diagonal elements (Eqs. (9) and (12)).

There are no gaps at $\mathbf{k}=\mathbf{k}^{*}$, when $\epsilon_{1}\left(\mathbf{k}^{*}\right)=\epsilon_{2}\left(\mathbf{k}^{*}\right)=$ $\epsilon_{3}\left(\mathbf{k}^{*}\right)=0$. In general, each of the equations $\epsilon_{i}(\mathbf{k})=0$ $(i=1,2$, and 3$)$ gives the line in the plane of $k_{x}$ and $k_{y}$. Therefore, it is only in rare case that three lines $\left(\epsilon_{i}(\mathbf{k})=\right.$ $0(i=1,2$, and 3$))$ intersect at the same point ${ }^{21,22}$. However, when the system has an inversion symmetry, i.e., $\mathrm{A}$ and $\mathrm{B}$ sublattices are equivalent, we get

$$
\epsilon_{3}(\mathbf{k})=0
$$

and there exist Dirac points where two lines $\left(\epsilon_{1}(\mathbf{k})=0\right.$ and $\left.\epsilon_{2}(\mathbf{k})=0\right)$ intersect. Hereafter, we study the system with inversion symmetry, i.e., Eq. (14) is satisfied.

As we have discussed, the next-nearest-neighbor hoppings appear only in $\epsilon_{0}(\mathbf{k})$ and $\epsilon_{3}(\mathbf{k})$. Therefore, the next-nearest-neighbor hoppings do not affect the existence and the location of the Dirac points in the system with inversion symmetry. They only affect the energy dispersion near the Dirac points, i.e. they may tilt a Dirac cone. Since we are interested in the location of the Dirac points and the topological phase transitions, we ignore the effects of the next-nearest-neighbor hoppings in this paper.

\section{TOPOLOGICAL NUMBER}

We define $\epsilon(\mathbf{k})$ and $\phi(\mathbf{k})$ by

$$
\begin{aligned}
& \epsilon_{1}(\mathbf{k})=\epsilon(\mathbf{k}) \cos (\phi(\mathbf{k})), \\
& \epsilon_{2}(\mathbf{k})=\epsilon(\mathbf{k}) \sin (\phi(\mathbf{k})) .
\end{aligned}
$$

Then the matrix given in Eq. (8) is written as

$$
\mathcal{E}_{\mathbf{k}}=\epsilon(\mathbf{k})\left(\cos (\phi(\mathbf{k})) \sigma_{1}+\sin (\phi(\mathbf{k})) \sigma_{2}\right),
$$

and it is diagonalized as

$$
U_{\mathbf{k}}^{-1} \mathcal{E}_{\mathbf{k}} U_{\mathbf{k}}=\epsilon(\mathbf{k}) \sigma_{3},
$$

where

$$
\begin{aligned}
U_{\mathbf{k}} & =e^{-i \frac{\phi(\mathbf{k})}{2} \sigma_{3}} e^{-i \frac{\pi}{4} \sigma_{2}} \\
& =\frac{1}{\sqrt{2}}\left(\begin{array}{cc}
e^{-i \frac{\phi(\mathbf{k})}{2}} & -e^{-i \frac{\phi(\mathbf{k})}{2}} \\
e^{i \frac{\phi(\mathbf{k})}{2}} & e^{i \frac{\phi(\mathbf{k})}{2}}
\end{array}\right) .
\end{aligned}
$$

(a)

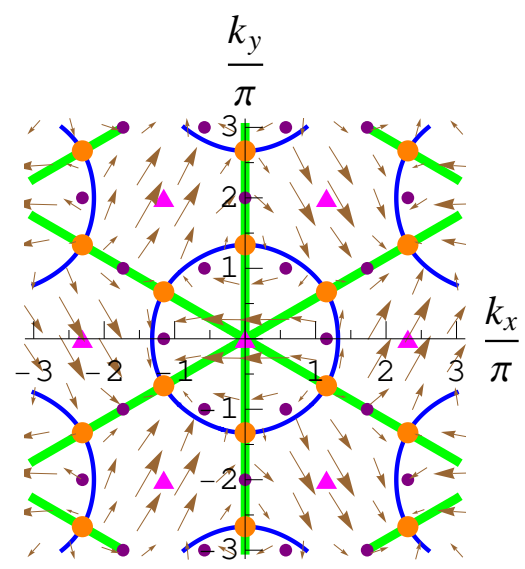

(b)

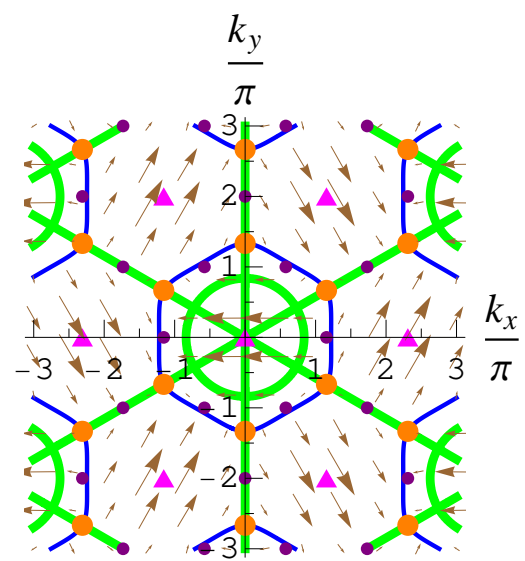

FIG. 2. (Color online) Lines for $\epsilon_{1}(\mathbf{k})=0$ (thin blue lines) and $\epsilon_{2}(\mathbf{k})=0$ (thick green lines). Parameters are $t_{a}=t_{b}=t_{c}=1$, $t_{3 a}=t_{3 b}=t_{3 c}=0$ in (a), and $t_{a}=t_{b}=t_{c}=1, t_{3 a}=$ $t_{3 b}=t_{3 c}=0.2$ in (b). Orange circles show $\mathbf{K}, \mathbf{K}^{\prime}$ and their equivalent points. Small purple circles are $\mathbf{M}_{i}(i=1,2$ and 3 ) and their equivalent points, and triangles are $\boldsymbol{\Gamma}$ and their equivalent points. Arrows show the vectors, $\left(\epsilon_{1}(\mathbf{k}), \epsilon_{2}(\mathbf{k})\right)$. Dirac points appear at the intersection points of the blue lines and the green lines, which are $\mathbf{K}$ and $\mathbf{K}^{\prime}$ in both figures. New green circular lines appear around $\mathbf{k}=0$, if $t_{3 a}>t_{a} / 8$ as shown in (b). The Dirac points are, however, only at the $\mathbf{K}$ and $\mathbf{K}^{\prime}$, if $t_{3 a}<t_{a} / 3$. The topological numbers are +1 and -1 for $\mathbf{K}$ and $\mathbf{K}^{\prime}$, respectively.

Therefore, the vectors,

$$
\Psi_{ \pm}(\mathbf{k})=\frac{1}{\sqrt{2}}\left(\begin{array}{c} 
\pm e^{-i \frac{\phi(\mathbf{k})}{2}} \\
e^{i \frac{\phi(\mathbf{k})}{2}}
\end{array}\right),
$$

are the eigenvectors of the matrix $\mathcal{E}_{\mathbf{k}}$ with eigenvalues $\pm \epsilon(\mathbf{k})$.

In Fig. 2, we plot the lines given by $\epsilon_{1}(\mathbf{k})=0$ (thin blue lines) and $\epsilon_{2}(\mathbf{k})=0$ (thick green lines) for $t_{a}=t_{b}=$ $t_{c}=1, t_{3 a}=t_{3 b}=t_{3 c}=0$ in (a) and $t_{a}=t_{b}=t_{c}=1$, 
$t_{3 a}=t_{3 b}=t_{3 c}=0.2 \mathrm{in}(\mathrm{b})$. The Dirac points are given by the intersection points of the blue lines and the green lines, which are at

$$
\begin{aligned}
\mathbf{K} & =\left(0, \frac{4 \pi}{3}\right), \\
\mathbf{K}^{\prime} & =\left(0,-\frac{4 \pi}{3}\right),
\end{aligned}
$$

and their equivalent points related by the reciprocal lattice vectors. We also plot the vector $\left(\epsilon_{1}(\mathbf{k}), \epsilon_{2}(\mathbf{k})\right)$ in Fig. 2. The Dirac points are the cores of the vortex for the vector $\left(\epsilon_{1}(\mathbf{k}), \epsilon_{2}(\mathbf{k})\right)$. The topological number is +1 and -1 at $\mathbf{K}$ and $\mathbf{K}^{\prime}$, respectively. Note that the configurations of the arrows have a topological number +1 , if they are clockwisely or counterclockwisely rotating flows around the core, or if all arrows are pointing out or into the cores, since $\phi(\mathbf{k})$ changes $+2 \pi$ when $\mathbf{k}$ goes counterclockwisely around the core. The configurations of the arrows have a topological number -1 , if they are pointing out the core in $\pm x$ direction and pointing into the core in the $\pm y$ direction, for example.

Since the eigenstates are given by $\phi(\mathbf{k})($ Eq. (20)), the topological number \pm 1 is related to the Berry phase of $\pm \pi$.

\section{MERGING DIRAC POINTS AT M OR $\Gamma$ IN THE BRILLOUIN ZONE}

When $\mathbf{k}^{*}$ is a Dirac points, $-\mathbf{k}^{*}$ is also a Dirac point if the system is invariant with respect to the time-reversal operation, which is always the case in this paper. Two Dirac points, which are exchanged by time-reversal operation, can merge only at the points $\mathbf{k}^{*}$ where $\mathbf{k}^{*}$ and $-\mathbf{k}^{*}$ are the same or are different by the reciprocal lattice vectors. These points are only four points in the Brillouin zone, $\mathbf{M}_{1}, \mathbf{M}_{2}, \mathbf{M}_{3}$, and $\mathbf{\Gamma}$,

$$
\begin{aligned}
\mathbf{M}_{1} & =\left(\frac{\sqrt{3} \pi}{3}, \pi\right), \\
\mathbf{M}_{2} & =\left(\frac{2 \sqrt{3} \pi}{3}, 0\right), \\
\mathbf{M}_{3} & =\left(\frac{\sqrt{3} \pi}{3},-\pi\right), \\
\mathbf{\Gamma} & =(0,0) .
\end{aligned}
$$

From the equation

$$
\epsilon_{1}\left(\mathbf{M}_{1}\right)=\epsilon_{2}\left(\mathbf{M}_{1}\right)=0,
$$

we obtain the condition for two Dirac points to merge at $\mathbf{M}_{1}$ as

$$
t_{a}+t_{b}-t_{c}=t_{3 a}+t_{3 b}+t_{3 c} \quad: \mathbf{M}_{1} .
$$

Similarly we obtain the conditions for two Dirac points to merge at $\mathbf{M}_{2}, \mathbf{M}_{3}$, and $\boldsymbol{\Gamma}$ as

$$
\begin{aligned}
-t_{a}+t_{b}+t_{c}=t_{3 a}+t_{3 b}+t_{3 c} & : \mathbf{M}_{2}, \\
t_{a}-t_{b}+t_{c}=t_{3 a}+t_{3 b}+t_{3 c} & : \mathbf{M}_{3},
\end{aligned}
$$

(a)

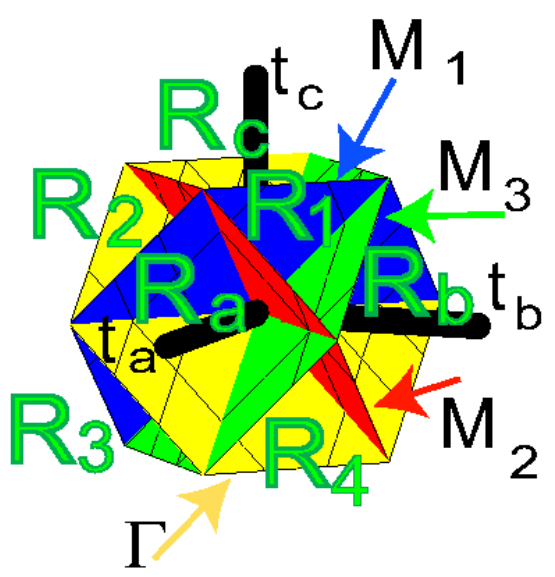

(b)

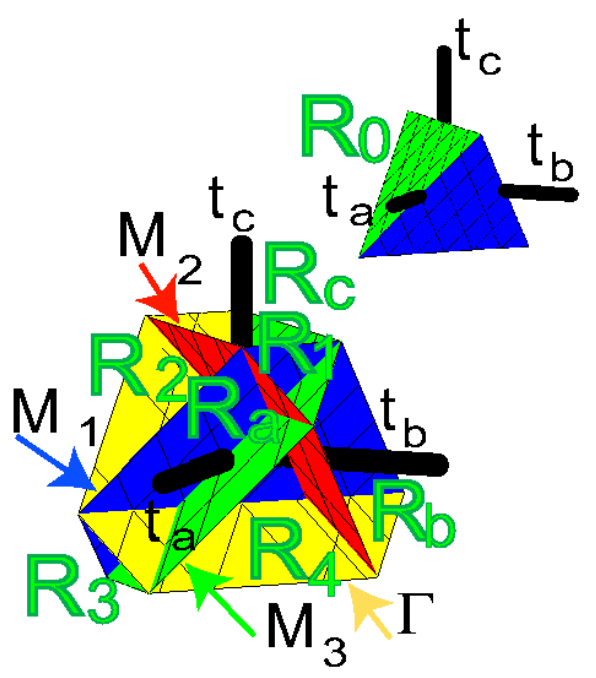

FIG. 3. (Color online) Planes given by Eqs. (28) - (31) in the parameter space of $t_{a}, t_{b}$ and $t_{c}$ in the case of (a) $t_{3 a}=t_{3 b}=t_{3 c}=0$ and (b) $t_{3 a}=t_{3 b}=t_{3 c}>0$. On the planes labeled by $M_{1}, M_{2}, M_{3}$ and $\Gamma$, two Dirac points merge at $\mathbf{M}_{1}, \mathbf{M}_{2}, \mathbf{M}_{3}$, and $\boldsymbol{\Gamma}$, respectively. The $3 \mathrm{D}$ space is divided into 14 regions, $R_{1}-R_{4}, R_{a}, R_{b}, R_{c}, R_{1}^{*}-R_{4}^{*}, R_{a}^{*}, R_{b}^{*}$, and $R_{c}^{*}$ when $t_{3 a}=t_{3 b}=t_{3 c}=0$. The regions, $R_{1}^{*}, R_{2}^{*}$ etc. are given by the inversion of $R_{1}, R_{2}$ etc.. When $t_{3 a}=t_{3 b}=t_{3 c} \neq 0$, a regular tetrahedral region $R_{0}$ appears around the origin, as shown in the inset of (b). Topological phase transitions occur and the number of the Dirac points changes by two when parameters intersect the planes, $M_{1}, M_{2}, M_{3}$ and $\Gamma$.

and

$$
-t_{a}-t_{b}-t_{c}=t_{3 a}+t_{3 b}+t_{3 c} \quad: \boldsymbol{\Gamma},
$$

respectively. We plot the planes given by Eqs. (28) - (31) in the parameter space in $t_{a}, t_{b}$ and $t_{c}$ in the cases of $t_{3 a}=t_{3 b}=t_{3 c}=0$ and $t_{3 a}=t_{3 b}=t_{3 c}>0$ in Fig. 3 . 


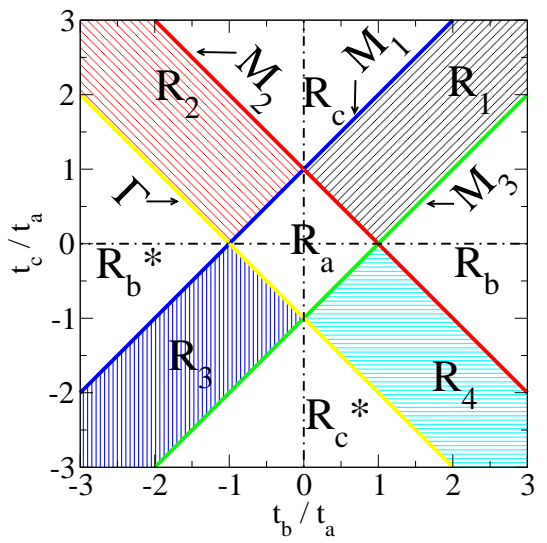

FIG. 4. (Color online) Phase diagram in the plane of $t_{b} / t_{a^{-}}$ $t_{c} / t_{a}$ for the tight-binding electrons on honeycomb lattice with direction-dependent nearest-neighbor hoppings $\left(t_{3 a}=\right.$ $\left.t_{3 b}=t_{3 c}=0\right)$.

The phase diagram in $t_{b} / t_{a}-t_{c} / t_{a}$ plane (Fig. 4) can be obtained as the intersection of the phase diagram in 3D plot (Fig. 3) and the plane of constant $t_{a}$, as shown in Fig. 5.

If $t_{3 a}+t_{3 b}+t_{3 c}=0$, the $t_{a}-t_{b}-t_{c}$ space is divided into 14 regions by four planes, Eqs. (28) - (31), as shown in Fig. 3 (a). There are two gapless Dirac points when parameters are in the regions labeled by $R_{1}, R_{2}, R_{3}$ and $R_{4}$ and their inversion regions, $R_{1}^{*}, R_{2}^{*}, R_{3}^{*}$ and $R_{4}^{*}$ in Fig. 3. The regions, $R_{1}, R_{2}, R_{3}$ and $R_{4}$, correspond to the shaded regions in Fig. 4, where two-dimensional plot of the phase diagram is shown in $t_{b} / t_{a}-t_{c} / t_{a}$ plane. On the planes and lines $\left(M_{1}, M_{2}, M_{3}\right.$ and $\left.\Gamma\right)$ in Fig. 3 and Fig. 4, topological phase transition occurs and two Dirac points merges at $\mathbf{M}_{1}, \mathbf{M}_{2}, \mathbf{M}_{3}$ or $\boldsymbol{\Gamma}$ in the Brillouin zone. The phase diagram for $t_{3 a}+t_{3 b}+t_{3 c}=0$ is the same as obtained previously ${ }^{7}$.

We show the 3D plot of the phase diagram in Fig. 3 (b) for $t_{3 a}+t_{3 b}+t_{3 c}>0$. Although it is invisible in the main figure in Fig. 3 (b), there is a regular tetrahedron region $R_{0}$ around the origin $(0,0,0)$ as shown in the inset in Fig. 3 (b). We plot the phase diagram in the plane of $t_{b} / t_{a}-t_{c} / t_{a}$ plane for $t_{a}=1$ and $t_{3 a}=t_{3 b}=t_{3 c}=0.19 \mathrm{in}$ Fig. 6. If the third-nearest-neighbor hoppings are finite, the number of the Dirac points can be larger than 2 and Dirac points can merge at the points other than $\mathbf{M}_{1}, \mathbf{M}_{2}$, $\mathbf{M}_{3}$ and $\boldsymbol{\Gamma}$. The topological phase transitions caused by these merging Dirac points are shown by the orange lines in Fig. 6, which we will discuss in Section VI.

The boundary lines $M_{1}$ and $M_{3}$ in $t_{b} / t_{a}-t_{c} / t_{a}$ plane move closer and the regions $R_{1}$ and $R_{2}$ becomes narrower, as $t_{3 a}+t_{3 b}+t_{3 c}$ becomes large, as seen in Fig. 5 (a). When $t_{3 a}+t_{3 b}+t_{3 c}=t_{a}$, the boundaries $\left(M_{1}\right.$ and $\left.M_{3}\right)$ in the $t_{b} / t_{a}-t_{c} / t_{a}$ plane overlap and the regions $R_{1}$ and $R_{3}$ disappear in the phase diagram in the $t_{b} / t_{a}-t_{c} / t_{a}$ plane. If $t_{3 a}+t_{3 b}+t_{3 c}>t_{a}, M_{1}$ and $M_{3}$ lines are exchanged and the new region $R_{0}$ appears, as seen in Fig. 5 (b). (a)

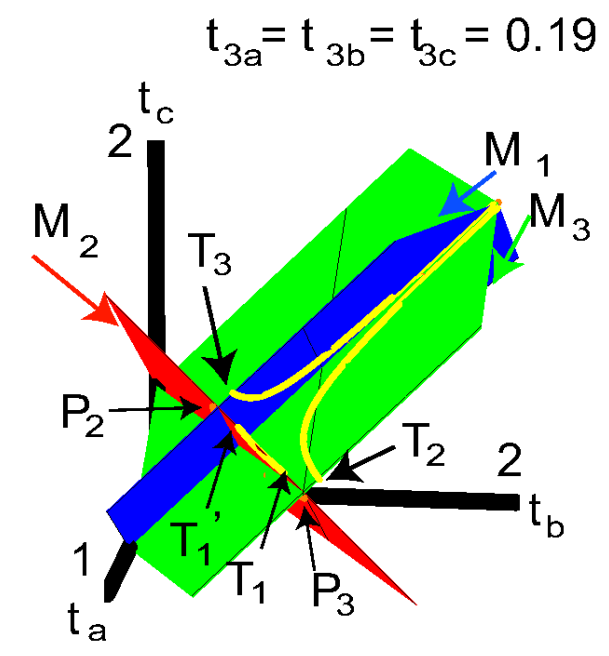

(b)

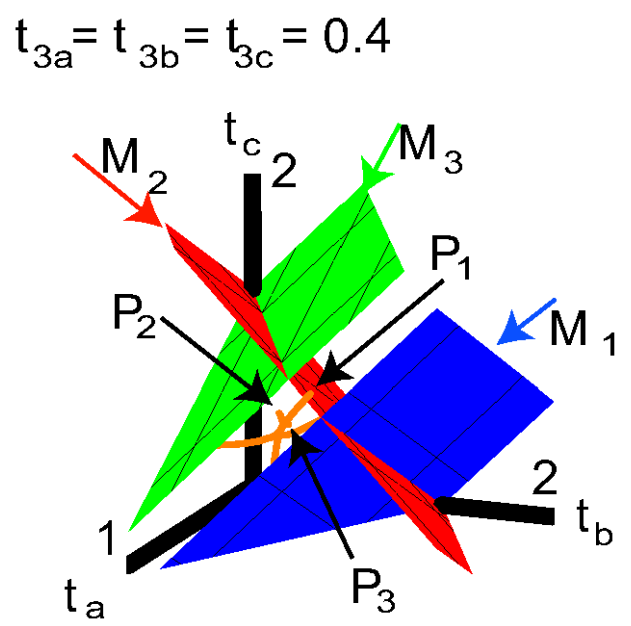

FIG. 5. (Color online) 3D plot of the phase diagrams for (a) $t_{3 a}=t_{3 b}=t_{3 c}=0.19$ and (b) 0.4 . The intersection with the plane $t_{a}=1$ is the phase diagram in Fig. 6 and Fig. 15 (b). Three Dirac points merge when parameters are at $\mathbf{P}_{1}, \mathbf{P}_{2}$, or $\mathbf{P}_{3}$, and $\mathbf{T}_{1}, \mathbf{T}_{1}^{\prime}, \mathbf{T}_{2}$, and $\mathbf{T}_{3}$ are tricritical points, which are discussed in Section VI and Appendix.

\section{MOVING DIRAC POINTS WITH ONLY NEAREST NEIGHBOR HOPPINGS}

In this section, only nearest neighbor hoppings are considered $\left(t_{a}^{\prime}=t_{b}^{\prime}=t_{c}^{\prime}=t_{a}^{\prime \prime}=t_{b}^{\prime \prime}=t_{c}^{\prime \prime}=0\right.$ and $\left.t_{3 a}=t_{3 b}=t_{3 c}=0\right)$. When $\left|t_{a}\right|,\left|t_{b}\right|$ and $\left|t_{c}\right|$ satisfy the "triangle inequality",

$$
\left|\frac{\left|t_{b}\right|}{\left|t_{a}\right|}-1\right| \leq \frac{\left|t_{c}\right|}{\left|t_{a}\right|} \leq \frac{\left|t_{b}\right|}{\left|t_{a}\right|}+1
$$




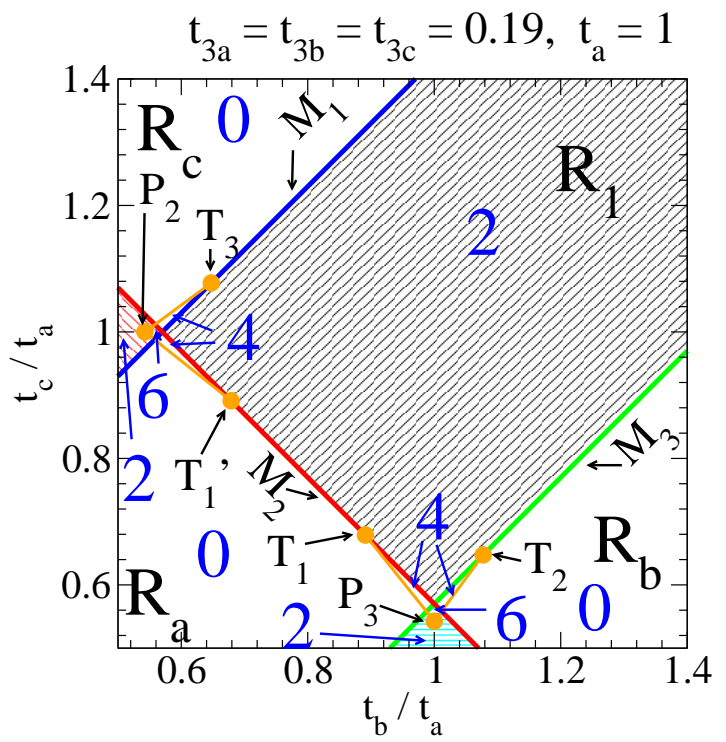

FIG. 6. (Color online) Phase diagrams in the $t_{b} / t_{a}-t_{c} / t_{a}$ plane for $t_{a}=1$ and $t_{3 a}=t_{3 b}=t_{3 c}=0.19$. When the thirdnearest-neighbor hoppings are finite, phase transition lines $M_{1}, M_{2}, M_{3}$ and $\Gamma$ are shifted and new transition lines appear, as discussed in Section VI and Appendix. The phase diagrams for other values of third-nearest-neighbor hoppings calculated numerically are show in Fig. 14 and Fig. 15. The numbers in the figures indicate the numbers of the Dirac points in the Brillouin zone.

there are two Dirac points at $\mathbf{k}= \pm \mathbf{k}^{*}$. The position of the Dirac points is obtained by the equations ${ }^{7,21,22,26}$

$$
\begin{aligned}
\cos \left(\mathbf{v}_{1} \cdot \mathbf{k}^{*}\right) & =\frac{t_{c}^{2}-t_{a}^{2}-t_{b}^{2}}{2 t_{a} t_{b}}, \\
\cos \left(\mathbf{v}_{2} \cdot \mathbf{k}^{*}\right) & =\frac{t_{b}^{2}-t_{a}^{2}-t_{c}^{2}}{2 t_{c} t_{a}}, \\
\cos \left(\left(\mathbf{v}_{1}-\mathbf{v}_{2}\right) \cdot \mathbf{k}^{*}\right) & =\frac{t_{a}^{2}-t_{b}^{2}-t_{c}^{2}}{2 t_{b} t_{c}} .
\end{aligned}
$$

Note that the third equation (Eq. (35)) is satisfied if the first and the second equations (Eqs. (33) and (34)) are satisfied and

$$
t_{b} t_{c} \sin \left(\mathbf{v}_{1} \cdot \mathbf{k}^{*}\right) \sin \left(\mathbf{v}_{2} \cdot \mathbf{k}^{*}\right)>0 .
$$

The condition, Eq. (32), is fulfilled in the regions $R_{1}, R_{2}$, $R_{3}, R_{4}, R_{1}^{*}, R_{2}^{*}, R_{3}^{*}$, and $R_{4}^{*}$, in Fig. 3 (a). We plot the trajectories of the Dirac points as $t_{c}$ is changed for $t_{a}=t_{b}=1$ in Fig. 7. When $t_{c}=1$ two Dirac points are located at $\mathbf{K}$ and $\mathbf{K}^{\prime}$ (up triangles and down triangles labeled by "1" in Fig. 7). The Dirac points move to $\mathbf{M}_{1}$ and the equivalent points as $t_{c}$ increases (blue broken lines and black lines in Fig. 7), and they merge at $\mathbf{M}_{1}$ and the equivalent points when $t_{c}=2$. Dirac points annihilate when $t_{c}>2$. Merging of two Dirac points causes a topological phase transition and this phase boundary is shown as $M_{1}$ in Fig. 3 and Fig. 4. On the other hand,

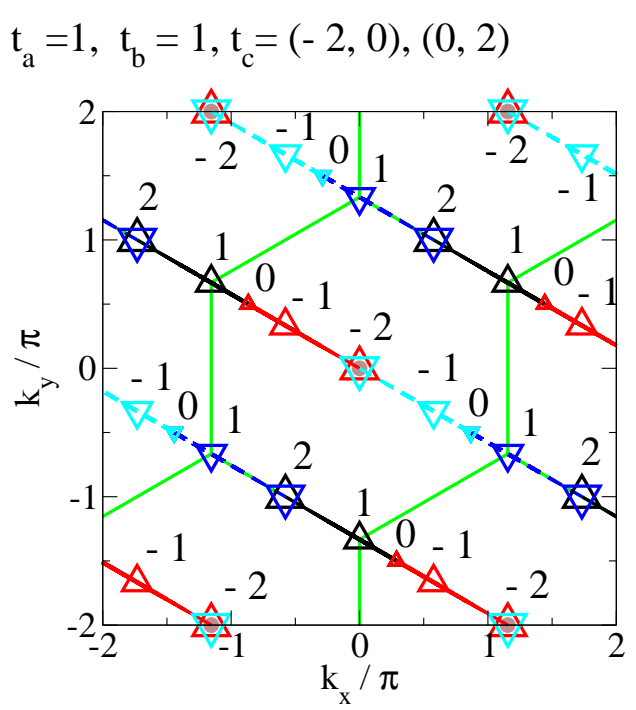

FIG. 7. (Color online) Locations and trajectories of the Dirac points as a function of $t_{c}$ with $t_{a}=t_{b}=1$ being fixed. Only nearest neighbor hoppings are considered. Solid and Broken lines are the trajectories of the Dirac points, which are converted by the space inversion each other. Up triangles and down triangles are the position of the Dirac points at $t_{c}$ indicated at the figure. When $t_{c}=2$ and $t_{c}=-2$, two Dirac points merge at the $M_{1}$ point $\left(\frac{\pi}{\sqrt{3}}, \pi\right)$ and the $\Gamma$ point $(0,0)$, respectively. When $t_{c}=0$ the system becomes onedimensional. Therefore, the smallest up and down triangles are not the Dirac points, but Dirac points approach these points as $t_{c} \rightarrow 0$.

when $t_{c}$ is decreased, Dirac points move in the opposite direction. Dirac points approach to the midpoint of $\mathbf{M}_{2}$ and $\mathbf{M}_{3}, \pm\left(\mathbf{M}_{2}+\mathbf{M}_{3}\right) / 2= \pm(\sqrt{3} \pi / 2,-\pi / 2)$. When $t_{c}=0$, the system becomes one-dimensional and two bands intersect on the line. The smallest triangles with the label " 0 " in Fig. 7 should be considered as a singular points for the location of Dirac points as $t_{c} \rightarrow 0$. As seen in Fig. 3 (a) and Fig. $4, t_{a}=t_{b}=1$ and $t_{c}=0$ is the line (in Fig. 3 (a)) or the point (in Fig. 4) of intersection for the boundary planes or lines of $M_{2}$ and $M_{3}$. When $t_{c}$ is changed to be negative, the parameters move into the region $R_{4}$ in Fig. 3 (a) and in Fig. 4 . Dirac points move on the cyan broken lines and the red lines in Fig. 7 and merge at $\boldsymbol{\Gamma}$ when $t_{c}=-2$.

Similarly, if we change $t_{a}$ with fixing $t_{b}=t_{c}=1$, Dirac points move on the vertical lines, i.e., the figure is rotated by 120 degree. In that case Dirac points merge at $\mathbf{M}_{2}$ and the equivalent points when $t_{a}=2$.

In Fig. 8 we plot a set of trajectories as $t_{c}$ is changed $\left(\left|t_{b}-t_{a}\right| \leq t_{c} \leq t_{b}+t_{a}\right)$ for several values of $t_{b} / t_{a}>0$. Dirac points are located in the triangular regions around $\mathbf{K}$ and $\mathbf{K}^{\prime}$, when the parameters $t_{a}, t_{b}$, and $t_{c}$ are in the $R_{1}$ region in Fig. 3. Similarly, we obtain that the Dirac points move in other triangular regions in Fig. 9 when $t_{a}$, 


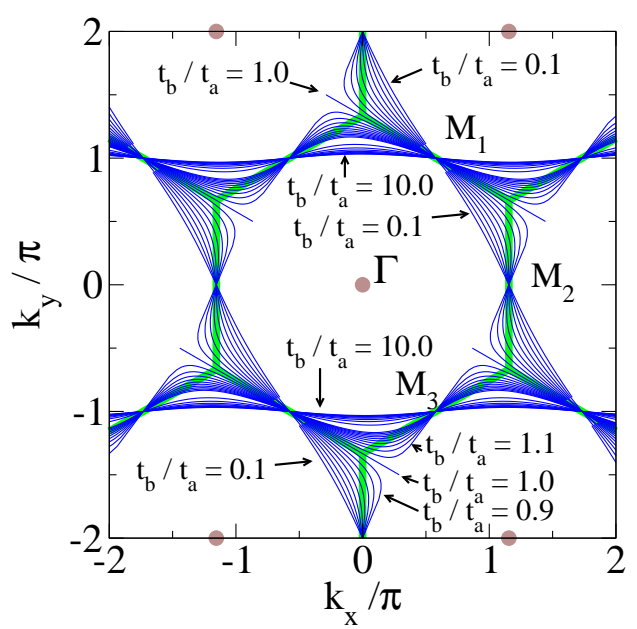

FIG. 8. (Color online) Locations of the Dirac points in the anisotropic honeycomb lattice with $t_{a}=1, t_{b}=$ $0.1,0.2, \cdots 0.9,1.0,1.1, \cdots 1.9,2.0,3.0,4.0, \cdots 9.0,10.0$. The lines are the trajectories of the Dirac points for $\left|t_{b}-t_{a}\right|<$ $t_{c}<t_{b}+t_{a}$ (region $R_{1}$ in Fig. 3)(a).

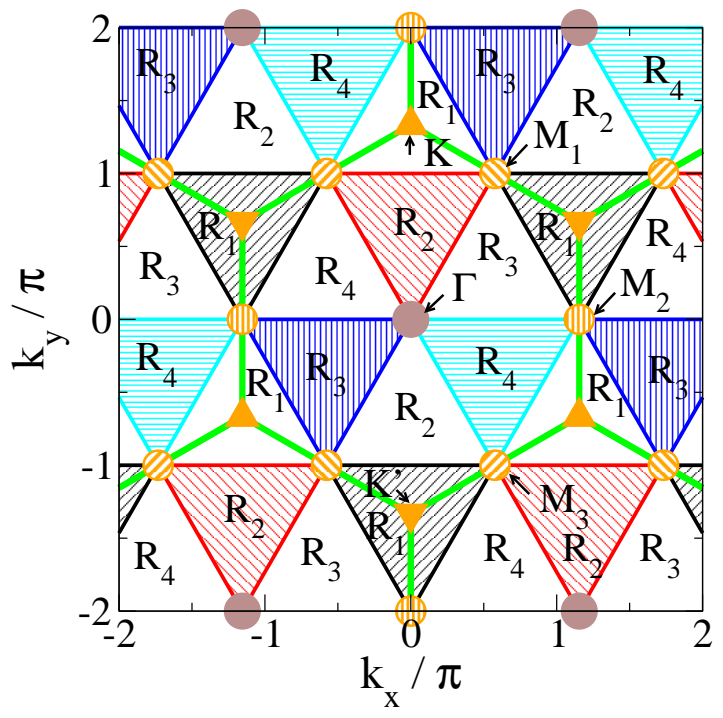

FIG. 9. (Color online) Divided regions of the moving Dirac points in the case of $\left(t_{a}, t_{b}, t_{c}\right)$ being in the regions of $R_{1}, R_{2}$, $R_{3}$ and $R_{4}$ in Fig. 3(a).

$t_{b}$, and $t_{c}$ are in $R_{2}, R_{3}$ or $R_{4}$ regions in Fig. 3. In Fig. 9 we plot the regions for the Dirac points when parameters $t_{a}, t_{b}$ and $t_{c}$ are in $R_{1}, R_{2}, R_{3}$ and $R_{4}$ in Fig. 3.

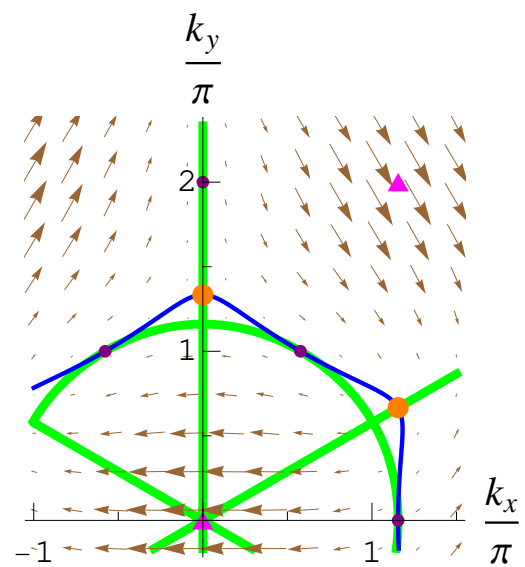

FIG. 10. (Color online) Lines for $\epsilon_{1}(\mathbf{k})=0$ (thin blue lines) and $\epsilon_{2}(\mathbf{k})=0$ (thick green lines) for $t_{a}=t_{b}=t_{c}=1, t_{3 a}=$ $t_{3 b}=t_{3 c}=1 / 3$. Green and Blue lines touch at $\mathbf{M}_{1}, \mathbf{M}_{2}$ and $\mathbf{M}_{3}$.

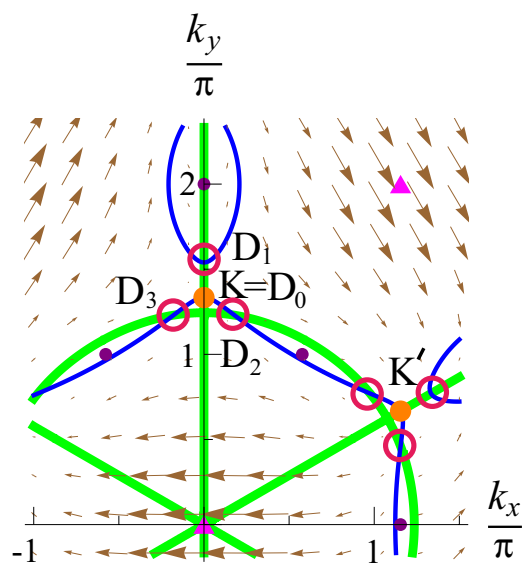

FIG. 11. (Color online) Lines for $\epsilon_{1}(\mathbf{k})=0$ (thin blue lines) and $\epsilon_{2}(\mathbf{k})=0$ (thick green lines) for $t_{a}=t_{b}=t_{c}=1$, $t_{3 a}=t_{3 b}=t_{3 c}=0.4$. Dirac points appear at $\mathbf{K}$ and $\mathbf{K}^{\prime}$ and additional points (red circles, $\mathbf{D}_{1}, \mathbf{D}_{2}$ and $\mathbf{D}_{3}$ ). The topological number is -1 and +1 for the additional three points around $\mathbf{K}$ and $\mathbf{K}^{\prime}$, respectively.

\section{DIRAC POINTS IN THE SYSTEM WITH THIRD-NEAREST-NEIGHBOR HOPPINGS}

When the third-nearest-neighbor hoppings are finite, more than two Dirac points are possible to exist. For simplicity we assume all hoppings, $t_{a}, t_{b}, t_{c}, t_{3 a}, t_{3 b}$, and $t_{3 c}$ are positive. Generalization to the negative hoppings is straightforward. 


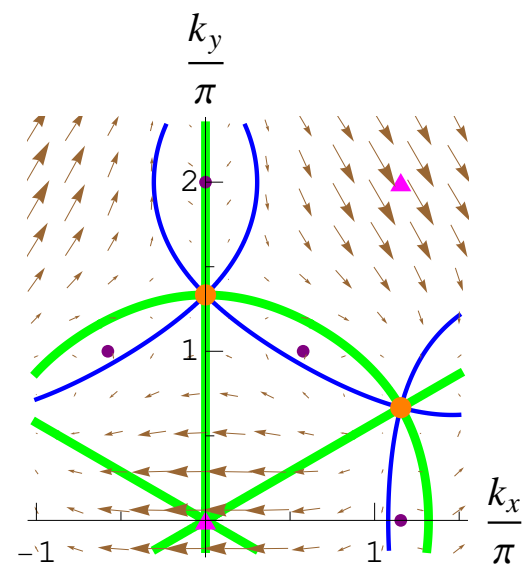

FIG. 12. (Color online) Lines for $\epsilon_{1}(\mathbf{k})=0$ (thin blue lines) and $\epsilon_{2}(\mathbf{k})=0$ (thick green lines) for $t_{a}=t_{b}=t_{c}=1$, $t_{3 a}=t_{3 b}=t_{3 c}=0.5$. Four Dirac points around $\mathbf{K}$ and $\mathbf{K}^{\prime}$ merge and the topological number becomes $\mp 2$ at $\mathbf{K}$ and $\mathbf{K}^{\prime}$, respectively.

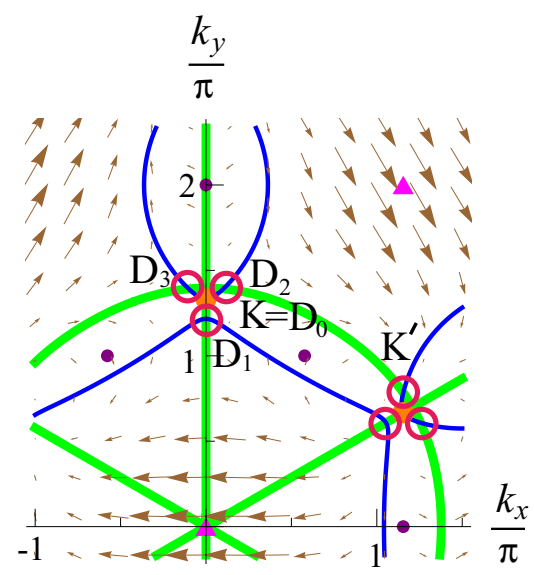

FIG. 13. (Color online) Lines for $\epsilon_{1}(\mathbf{k})=0$ (thin blue lines) and $\epsilon_{2}(\mathbf{k})=0$ (thick green lines) for $t_{a}=t_{b}=t_{c}=1, t_{3 a}=$ $t_{3 b}=t_{3 c}=0.6$. Dirac points locate at the intersection points of green lines and blue lines, i.e., $\mathbf{K}, \mathbf{K}^{\prime}, \mathbf{D}_{1}, \mathbf{D}_{2}$ and $\mathbf{D}_{3}$.

\section{A. Symmetric case $\left(C_{6 v}\right)$}

In this subsection we discuss the symmetric system,

$$
\begin{aligned}
t & \equiv t_{a}=t_{b}=t_{c}, \\
t_{3} & \equiv t_{3 a}=t_{3 b}=t_{3 c} .
\end{aligned}
$$

Bena and Simon ${ }^{15}$ have studied this model. They have shown that new Dirac points appear when $t_{3}>t / 3$. As shown in Fig. 2, the blue lines, which is given by $\epsilon_{1}(\mathbf{k})=$ 0 , and the green lines, which is given by $\epsilon_{2}(\mathbf{k})=0$, have intersection points only at $\mathbf{K}$ and $\mathbf{K}^{\prime}$, when $t_{3}<t / 3$. Derivation is given in Appendix A. The blue lines and the green lines touch at $\mathbf{M}_{1}, \mathbf{M}_{2}$ and $\mathbf{M}_{3}$, when $t_{3}=t / 3$ (Fig. 10). We perform power series expansions of $\epsilon_{1}(\mathbf{k})$ and $\epsilon_{2}(\mathbf{k})$ about $\mathbf{M}_{2}$ and obtain

$$
\begin{aligned}
& \epsilon_{1}(\mathbf{k}) \approx+t\left(k_{x}-\frac{2 \sqrt{3} \pi}{3}-\frac{1}{24} k_{y}^{2}\right), \\
& \epsilon_{2}(\mathbf{k}) \approx \frac{\sqrt{3}}{3} t\left(k_{x}-\frac{2 \sqrt{3} \pi}{3}+\frac{1}{8} k_{y}^{2}\right),
\end{aligned}
$$

when $t_{3}=t / 3$. This means that merged Dirac point exists at $\mathbf{M}_{2}$ when $t_{3}=t / 3$ and the energy is given by

$\sqrt{\left(\epsilon_{1}(\mathbf{k})\right)^{2}+\left(\epsilon_{2}(\mathbf{k})\right)^{2}} \approx t \sqrt{\frac{4}{3}\left(k_{x}-\frac{2 \sqrt{3} \pi}{3}\right)^{2}+\left(\frac{k_{y}^{2}}{12}\right)^{2}}$,

i.e., the energy is proportional to $\left|k_{x}-k_{x}^{*}\right|$ in one direction and proportional to $\left(k_{y}-k_{y}^{*}\right)^{2}$ in other direction. The merged Dirac points, which have these energy dispersion, are called semi-Dirac points ${ }^{27}$. When the semiDirac points exit, density of states is proportional to the square root of the energy ${ }^{7}$

$$
D(\epsilon) \propto \sqrt{\epsilon},
$$

and the energy of the Landau levels varies as $B^{2 / 3}$ [Ref. 28].

When $t_{3}$ is larger than $t / 3$, each touching point of blue and green lines changes to a pair of the intersection points, i.e., each semi-Dirac point separate into two Dirac points with topological number \pm 1 , and there exist eight Dirac points, as shown by $\mathbf{D}_{1}, \mathbf{D}_{2}$ and $\mathbf{D}_{3}$ around $\mathbf{K}\left(=\mathbf{D}_{0}\right)$ in Fig. 11. As $t_{3} / t$ becomes large, two Dirac points stay at $\mathbf{K}$ and $\mathbf{K}^{\prime}$ and other Dirac points move from $\mathbf{M}_{1}, \mathbf{M}_{2}$ and $\mathbf{M}_{3}$ to $\mathbf{K}$ and $\mathbf{K}^{\prime}$. Three Dirac points moving to $\mathbf{K}$ for $1 / 3<t_{3} / t<1 / 2\left(\mathbf{D}_{1}, \mathbf{D}_{2}\right.$ and $\mathbf{D}_{3}$ in Fig. 11) have the topological number -1 and three Dirac points moving to $\mathbf{K}^{\prime}$ have topological number +1 . When $t_{3}=t / 2$, four Dirac points merge at $\mathbf{K}$ and $\mathbf{K}^{\prime}$. In that case two blue lines and two green lines intersect at $\mathbf{K}$ and $\mathbf{K}^{\prime}$, as shown in Fig. 12. The merged Dirac points at $\mathbf{K}$ and $\mathbf{K}^{\prime}$ have topological number -2 and +2 , respectively $(1 \times(+1)+3 \times(-1)=-2$ at $\mathbf{K}$, for example). The topological number at $\mathbf{K}$ can be also obtained as follows. For $\left(k_{x}, k_{y}\right) \approx \mathbf{K}=(0,4 \pi / 3)$, we write

$$
\begin{aligned}
k_{x} & =\kappa \cos (\theta), \\
k_{y}-\frac{4}{3} \pi & =\kappa \sin (\theta) .
\end{aligned}
$$

When $t_{3}=t / 2$, we obtain

$$
\begin{aligned}
& \epsilon_{1}(\mathbf{k}) \approx \frac{3}{8} t \kappa^{2} \cos (2 \theta) \\
& \epsilon_{2}(\mathbf{k}) \approx-\frac{3}{8} t \kappa^{2} \sin (2 \theta) .
\end{aligned}
$$

Comparing these equations with Eqs. (15) and (16), we obtain

$$
\phi(\mathbf{k})=-2 \theta,
$$


and the topological number of the vector $\left(\epsilon_{1}(\mathbf{k}), \epsilon_{1}(\mathbf{k})\right)$ at $\mathbf{K}$ is -2 . The energy at this point is obtained as

$$
\sqrt{\left(\epsilon_{1}(\mathbf{k})\right)^{2}+\left(\epsilon_{2}(\mathbf{k})\right)^{2}} \approx \frac{3}{8} t\left(k_{x}^{2}+\left(k_{y}-\frac{4}{3} \pi\right)^{2}\right),
$$

which has been obtained by Bena and Simon ${ }^{15}$. In this case the density of states is constant near $\epsilon \approx 0$.

When $t_{3}>t / 2$, eight Dirac points appear again. Two Dirac points are at $\mathbf{K}$ and $\mathbf{K}^{\prime}$ having topological number +1 and -1 , respectively. There exist three Dirac points with topological number -1 around $\mathbf{K}$ and three Dirac points with topological number +1 around $\mathbf{K}^{\prime}$. They form the equilateral triangles as in the case of $t / 3<$ $t_{3}<t / 2$, but the triangles are upside-down, as shown in Fig. 13 for $t_{3}=0.6 t$.

\section{B. direction-dependent hoppings with third-nearest neighbor hoppings}

If the hoppings depend on the direction, the phase diagram becomes much richer. Even if the hoppings between the third-nearest-neighbor sites are small, there appear new phases as shown in Fig. 6. Two Dirac points can merge at the point other than $\mathbf{M}_{1}, \mathbf{M}_{2}, \mathbf{M}_{3}$, and $\boldsymbol{\Gamma}$. When a pair of the Dirac points merge at $\mathbf{k}^{*}$, which are neither $\mathbf{M}_{1}, \mathbf{M}_{2}, \mathbf{M}_{3}$ nor $\boldsymbol{\Gamma}$, in the Brillouin zone, other pair should merge at $-\mathbf{k}^{*}$ simultaneously, since we study the system with time-reversal symmetry. Therefore, when the parameters are moved across these phase boundary lines (orange lines in Fig. 6 and curved lines in Fig. 14 and Fig. 15), the number of the Dirac points changes by four, i.e., $0 \leftrightarrow 4,2 \leftrightarrow 6$ and $4 \leftrightarrow 8$.

When the system has the reflection symmetry $\left(C_{2 v}\right.$ symmetry) we can obtain the analytical expressions for some special points $\left(\mathbf{P}_{1}, \mathbf{P}_{2}, \mathbf{P}_{3}, \mathbf{Q}_{1}, \mathbf{Q}_{2}\right.$, and $\left.\mathbf{Q}_{3}\right)$ in the phase diagram (see Appendix B). At $\mathbf{P}_{1}, \mathbf{P}_{2}$ and $\mathbf{P}_{3}$ in the phase diagram in $t_{b} / t_{a}-t_{c} / t_{a}$ plane, three Dirac points merge.

There exist the tricritical points, at which three phases with zero, two and four Dirac points terminate, in the parameter space in $t_{b}$ and $t_{c}$, which we label as $\mathbf{T}_{1}, \mathbf{T}_{1}^{\prime}$, $\mathbf{T}_{2}$ and $\mathbf{T}_{3}$.

In order to make the discussion simpler, we assume the third-nearest-neighbor hoppings are independent of the direction, i.e., we take $t_{3} \equiv t_{3 a}=t_{3 b}=t_{3 c}$. We plot $\mathbf{P}_{1}, \mathbf{P}_{2}, \mathbf{P}_{3}, \mathbf{T}_{1}, \mathbf{T}_{1}^{\prime}, \mathbf{T}_{2}$ and $\mathbf{T}_{3}$ as lines in the $3 \mathrm{D}$ plot in $t_{a}-t_{b}-t_{c}$ space in Fig. 5 . In the $t_{b}-t_{c}$ plane at $t_{a}=1$, these points are obtained as

$$
\begin{aligned}
& \mathbf{P}_{1}=\left(\frac{2 t_{3}^{3 / 2}}{\sqrt{3 t_{3}-1}}, \frac{2 t_{3}^{3 / 2}}{\sqrt{3 t_{3}-1}}\right), \\
& \mathbf{P}_{2}=\left(3 t_{3}-4 t_{3}^{3}, 1\right),
\end{aligned}
$$

and

$$
\mathbf{P}_{3}=\left(1,3 t_{3}-4 t_{3}^{3}\right)
$$

(a)

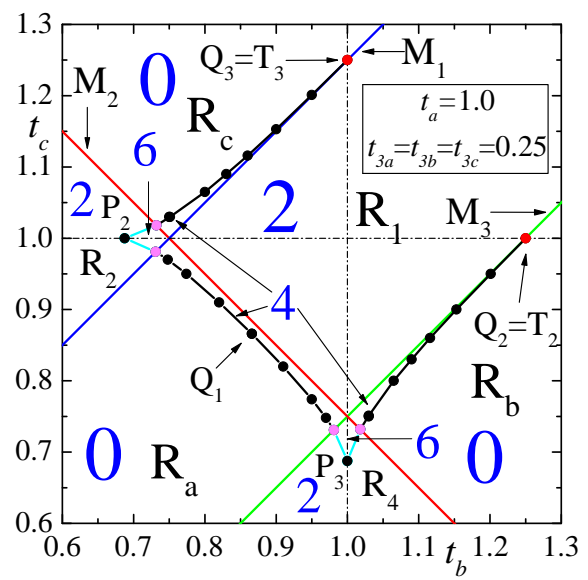

(b)

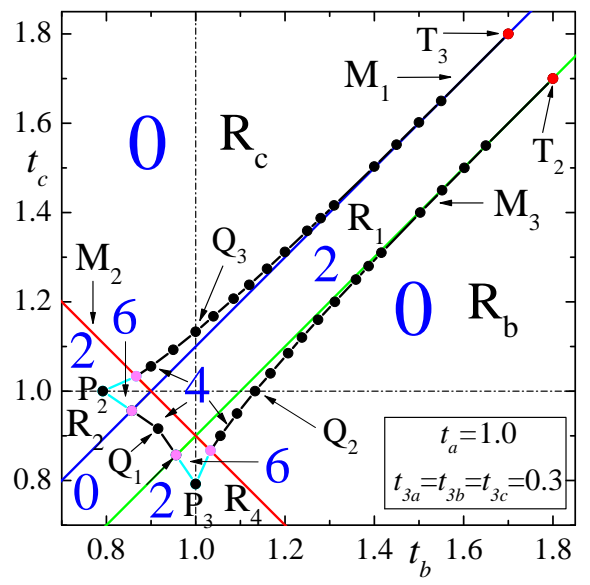

(c)

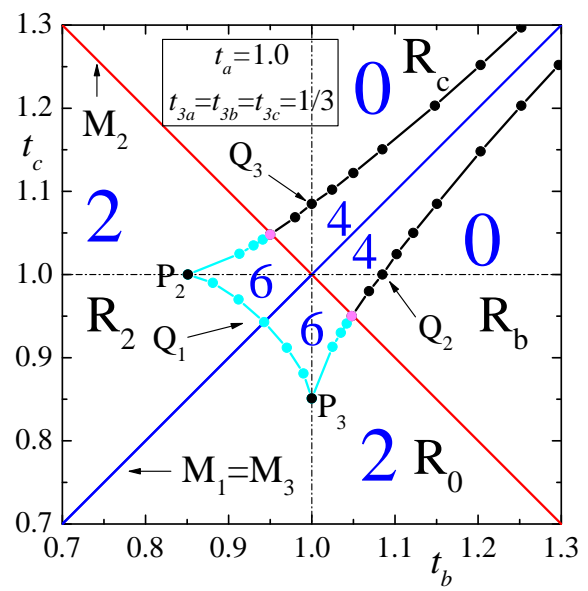

FIG. 14. (Color online) Phase diagram in the $t_{b}-t_{c}$ plane obtained numerically at $t_{a}=1$ and $t_{3 a}=t_{3 b}=t_{3 c}=0.25$, 0.3 , and $1 / 3$. The numbers of the Dirac points in the Brillouin zone is shown in the figure.

Note that while $\mathbf{P}_{1}$ exists only for $t_{3}>1 / 3, \mathbf{P}_{2}$ and $\mathbf{P}_{3}$ exist for any value of $t_{3} \neq 0$. The points $\mathbf{Q}_{1}, \mathbf{Q}_{2}$ and $\mathbf{Q}_{3}$ 
(a)

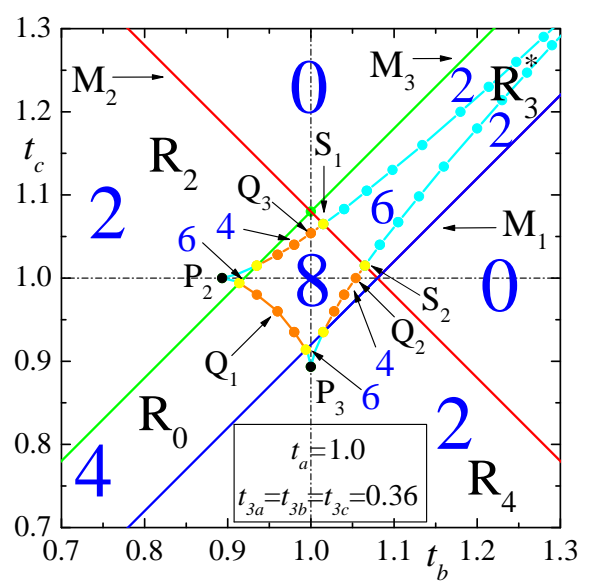

(b)

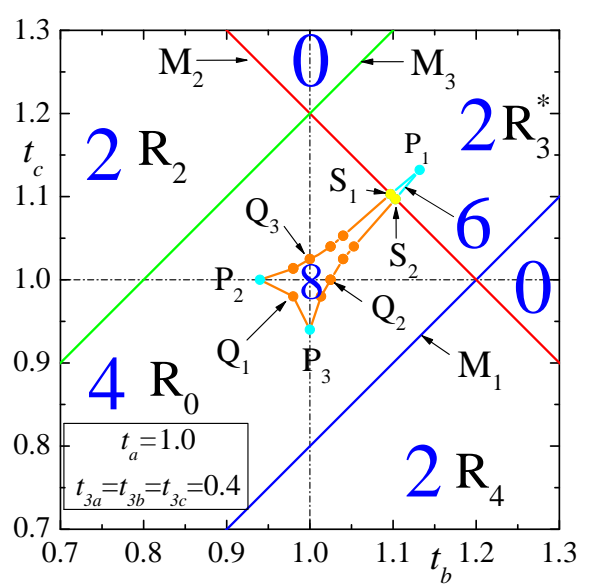

(c)

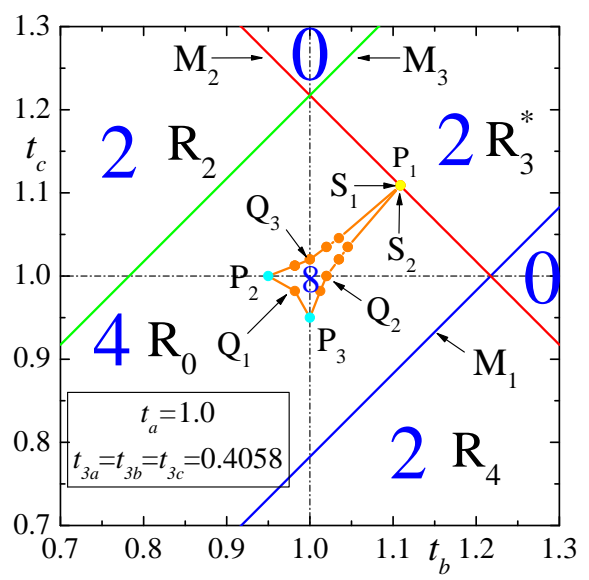

FIG. 15. (Color online) Phase diagram in the $t_{b}-t_{c}$ plane at $t_{a}=1$ and $t_{3 a}=t_{3 b}=t_{3 c}=0.36,0.4$ and 0.4058. The numbers in the figure indicate the number of the Dirac points in the Brillouin zone.

are given in Appendix B 2. We find that $\mathbf{Q}_{1}$ exists when $t_{3}>1 / 5$, and $\mathbf{Q}_{2}$ and $\mathbf{Q}_{3}$ exist when $t_{3}>1 / 4$.

The tricritical points are given as

$$
\begin{aligned}
& \mathbf{T}_{1}=\left(\frac{1+3 t_{3}}{2}+\frac{1+t_{3}}{2} \sqrt{\frac{1-5 t_{3}}{1+3 t_{3}}},\right. \\
&\left.\frac{1+3 t_{3}}{2}-\frac{1+t_{3}}{2} \sqrt{\frac{1-5 t_{3}}{1+3 t_{3}}}\right), \\
& \mathbf{T}_{1}^{\prime}=\left(\left(\mathbf{T}_{1}\right)_{y},\left(\mathbf{T}_{1}\right)_{x}\right), \\
& \mathbf{T}_{2}=\left(\frac{1-3 t_{3}}{2}+\frac{1-t_{3}}{2} \sqrt{\frac{1+5 t_{3}}{1-3 t_{3}}},\right. \\
&\left.\frac{-1+3 t_{3}}{2}+\frac{1-t_{3}}{2} \sqrt{\frac{1+5 t_{3}}{1-3 t_{3}}}\right),
\end{aligned}
$$

and

$$
\mathbf{T}_{3}=\left(\left(\mathbf{T}_{2}\right)_{y},\left(\mathbf{T}_{2}\right)_{x}\right) .
$$

Note that $\mathbf{T}_{1}$ and $\mathbf{T}_{1}^{\prime}$ exist when $0<t_{3}<1 / 5$, and $\mathbf{T}_{2}$ and $\mathbf{T}_{3}$ exist when $0<t_{3}<1 / 3$.

The phase transition lines connections $\mathbf{P}_{i}, \mathbf{Q}_{i}$ and $\mathbf{T}_{i}$ are obtained numerically and shown in Figs. 14 and 15 for $t_{3}=0.25,0.3,1 / 3,0.36,0.4$ and 0.4058 . The numbers of the Dirac points in the Brillouin zone changes by 2 when the phases are divided by the straight line and it changes by 4 when the phases are separated by the curved line.

In Fig. 16 we show the regions where $\mathbf{P}_{i}$ and $\mathbf{Q}_{i}$ locate. We plot the $t_{3}$ dependences of the coordinates of $\mathbf{P}_{i}, \mathbf{Q}_{i}$ and $\mathbf{T}_{i}$ in Fig. 17.

At the tricritical points, four Dirac points with topological numbers, $+1,+1,-1$ and -1 , merge at one of $\mathbf{M}_{i}$ in the Brillouin zone.

The density of states are calculated in Appendix D. We obtain

$$
D(\epsilon) \propto \begin{cases}|\epsilon| & \text { at Dirac point } \\ \sqrt{|\epsilon|} & \text { when two Dirac points merge } \\ & \text { (semi-Dirac point) } \\ |\epsilon|^{\frac{1}{3}} & \text { when three Dirac points merge } \\ & \mathbf{P}_{1}, \mathbf{P}_{2}, \mathbf{P}_{3} \\ |\epsilon|^{\frac{1}{4}} & \text { at tricritical point } \\ & \mathbf{T}_{1}, \mathbf{T}_{1}^{\prime}, \mathbf{T}_{2}, \mathbf{T}_{3} .\end{cases}
$$

Note that although the topological number of the merged point of three Dirac points is the same as that of the ordinary Dirac point, energy dependences of the density of states are different.

In Fig. 18 we show how Dirac points move when $t_{a}$ is changed when other parameters are taken as $t_{b}=t_{c}=1$ and $t_{3 a}=t_{3 b}=t_{3 c}=0.4$. The $3 \mathrm{D}$ plots of the energy are given in Fig.19 and Fig.20. When $t_{a}=1$, Dirac points are locates at $\mathbf{K}$ and apexes of the regular triangle (black circles in Fig. 18. See also Fig. 11.). As $t_{a}$ becomes larger, two Dirac points on the $k_{y}$ axis comes closer and merge when $t_{a}=41 / 40=1.025$ (this set of parameters corresponds to $\mathbf{Q}_{2}$ or $\mathbf{Q}_{3}$ in Fig. 15 (b) with exchanging $t_{a}$ and $t_{b}\left(\right.$ or $\left.\left.t_{c}\right)\right)$. These Dirac points disappear when $t_{a}>$ 


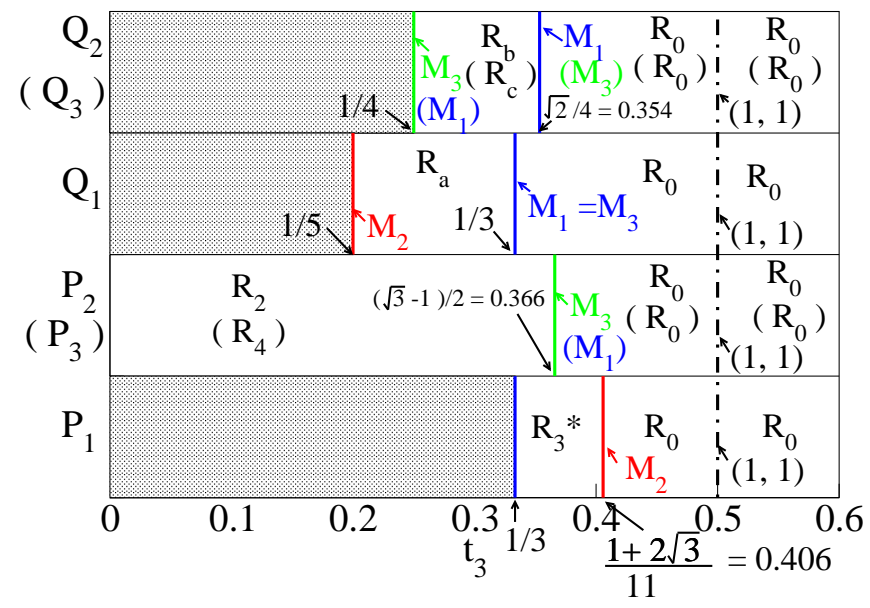

FIG. 16. (Color online) Locations of the parameters for three Dirac points to merge $\left(\mathbf{P}_{1}, \mathbf{P}_{2}\right.$ and $\left.\mathbf{P}_{3}\right)$ and for two Dirac points to merge in the axisymmetric case $\left(\mathbf{Q}_{1}, \mathbf{Q}_{2}\right.$ and $\left.\mathbf{Q}_{3}\right)$. When $t_{3}=0.5$ all points are at $(1,1)$. They do not exist in the shaded region $\left(0<t_{3}<1 / 5\right.$ for $\mathbf{Q}_{1}, 0<t_{3}<1 / 4$ for $\mathbf{Q}_{2}$ and $\mathbf{Q}_{3}$, and $0<t_{3}<1 / 3$ for $\left.\mathbf{P}_{1}\right)$. The regions labeled as $R_{a}, R_{b}, R_{c}, R_{0}, R_{2}, R_{4}$ and $R_{3}^{*}$, are those shown in Fig. 3 . The vertical lines labeled as $M_{1}, M_{2}$ and $M_{3}$ show that $\mathbf{P}_{i}$ and $\mathbf{Q}_{i}(i=1,2$, and 3$)$ are on the boundaries $\left(M_{1}, M_{2}\right.$ and $M_{3}$, respectively).

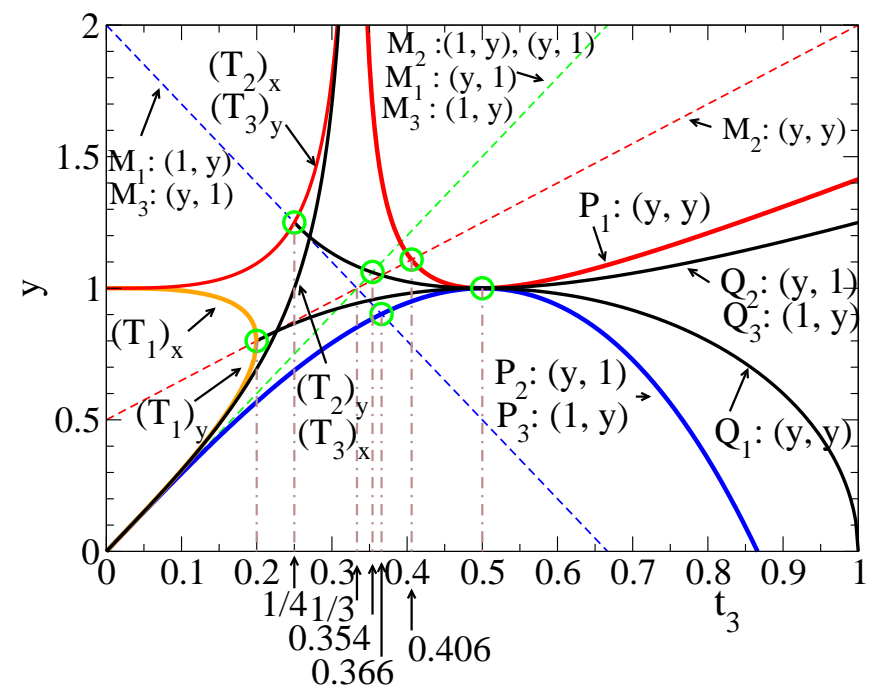

FIG. 17. (Color online) Coordinates of the special points in the phase diagram in $t_{b} / t_{a}-t_{c} / t_{a}$ plane as a function of $t_{3}$ $\left(t_{3}=t_{3 a}=t_{3 b}=t_{3 c}\right.$ and $\left.t_{a}=1\right)$. At $\mathbf{P}_{1}, \mathbf{P}_{2}$ and $\mathbf{P}_{3}$ (Eqs. (49), (50), and (51)), three Dirac points merge, and at $\mathbf{Q}_{1}, \mathbf{Q}_{2}$ and $\mathbf{Q}_{3}$ (Eqs. (B16), (B17), and (B18)), two Dirac points merge on the symmetric line $\left(k_{x}=0\right)$ in the Brillouin zone.

1.025. Other Dirac points move to $\mathbf{M}_{1}$ and $\mathbf{M}_{3}$ and they merge when $t_{a}=1.2$. When $t_{a}$ becomes smaller, three of the four Dirac points near $\mathbf{K}$ merge when $t_{a}=0.944$ (this set of parameters corresponds to $\mathbf{P}_{2}$ or $\mathbf{P}_{3}$ in Fig. 15 (b),

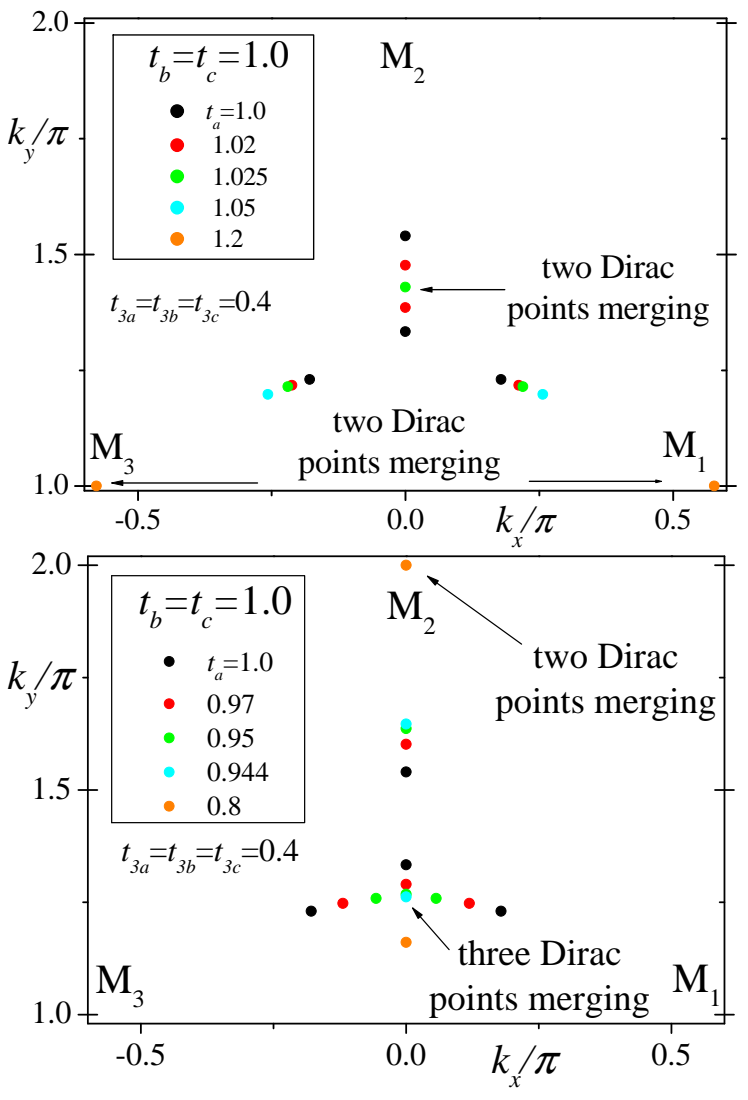

FIG. 18. (Color online) The moving Dirac points by changing $t_{a}$ with fixed $t_{b}=t_{c}=1$ and $t_{3 a}=t_{3 b}=t_{3 c}=0.4$.

see also Fig. 21) and one Dirac points remains after three Dirac points merge $\left(t_{a}<0.944\right)$. Another one of the four Dirac points move to $\mathbf{M}_{2}$ and merge when $t_{a}=0.8$.

\section{CONCLUSIONS}

We study the Dirac points of the electrons on the honeycomb lattice with up-to third-nearest neighbor hoppings. Since the next-nearest-neighbor hoppings do not affect the location of the Dirac points, we study the location of the Dirac points and the phase diagram in the parameter space in the direction-dependent nearestneighbor hoppings $\left(t_{a}, t_{b}\right.$ and $\left.t_{c}\right)$ and the third-nearestneighbor hoppings $\left(t_{3 a}, t_{3 b}\right.$ and $\left.t_{3 c}\right)$. Dirac points are obtained as the intersection points of two kinds of lines, which are given by $\epsilon_{1}(\mathbf{k})=0$ and $\epsilon_{2}(\mathbf{k})=0$. Dirac points move from $\mathbf{K}$ and $\mathbf{K}^{\prime}$, where Dirac points exist if hoppings are independent of the direction. We obtain the trajectories of the Dirac points as the directiondependent hoppings are changed. The topological phase transitions occur when Dirac points merge and disappear. We obtain the phase diagram in the parameter space of the direction-dependent hoppings. In each phase there are $0,2,4,6$, or 8 Dirac points, half of which have the topological number +1 and the other half have 

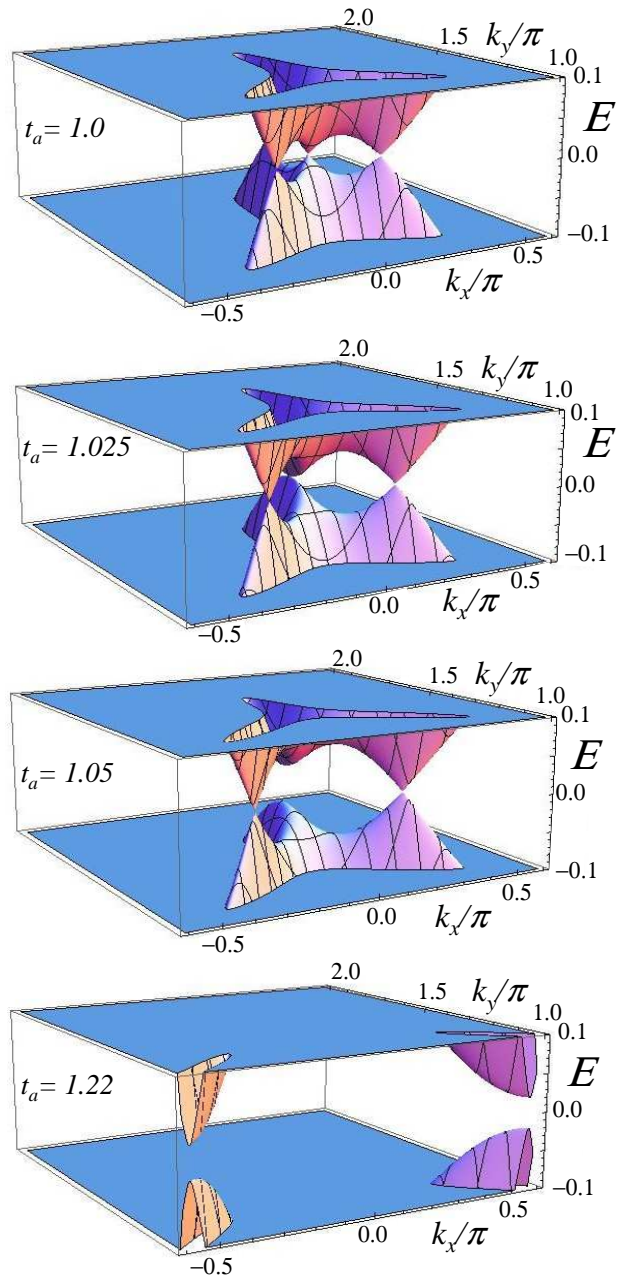

FIG. 19. (Color online) 3D plot of the energy as a function of the wave number for the parameters in Fig.18 $\left(t_{a} \geq 1\right)$.

the topological number -1 . The number of the Dirac points in the Brillouin zone changes by 2 when two Dirac points merge at $\mathbf{M}_{1}, \mathbf{M}_{2}, \mathbf{M}_{3}$, or $\boldsymbol{\Gamma}$, and it changes by 4 when two pairs of Dirac points merge at other points in the Brillouin zone, $\pm \mathbf{k}^{*}$. When parameters are at $\mathbf{P}_{1}$, $\mathbf{P}_{2}$ or $\mathbf{P}_{3}$, three Dirac points with the topological number $+1,-1$, and -1 (or $-1,+1$ and +1 ) merge. Four Dirac points with topological number $+1,-1,-1$, and $-1(-1,+1,+1$, and +1$)$ merge at $\mathbf{K}\left(\mathbf{K}^{\prime}\right)$ only when $t_{a}=t_{b}=t_{c}=2 t_{3 a}=2 t_{3 b}=2 t_{3 c}$. We also obtain that there are tricritical points $\mathbf{T}_{1}, \mathbf{T}_{1}^{\prime}, \mathbf{T}_{2}$ and $\mathbf{T}_{3}$, where three phases with 0,2 and 4 Dirac points terminate. There are no parameter regions where two Dirac points with the same topological number merge.

The density of states $D(\epsilon)$ for $\epsilon \approx 0$ is proportional to $|\epsilon|, \sqrt{\epsilon}, \epsilon^{1 / 3}, \epsilon^{1 / 4}$, and constant at the Dirac point, the merged point of two Dirac points (with topological number \pm 1 and $\mp 1$ ), the merged point of three Dirac points (two of them have the topological number \pm 1 and the other has the topological number $\mp 1$ ), the tricritical point, and the merged point of four Dirac points (three

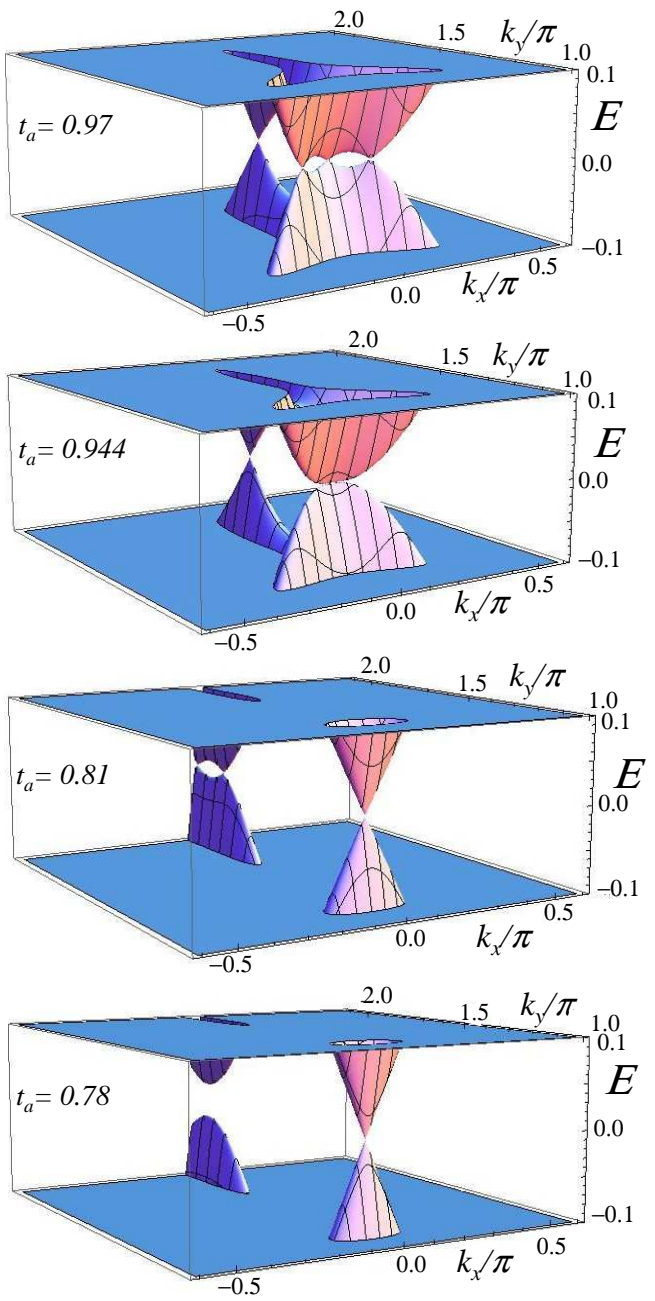

FIG. 20. (Color online) 3D plot of the energy as a function of the wave number for the parameters in Fig.18 $\left(t_{a}<1\right)$.

of then have the topological number \pm 1 and the other has the topological number $\mp 1$ ), respectively.

In order to realize these topological phase transitions, only small third-nearest-neighbor hoppings are necessary, if the anisotropy is sufficiently large. The thirdnearest-neighbor hoppings in monolayer graphene are estimated to be finite (about $0.1 t)^{20}$. On the other hand, if there are large third-nearest-neighbor hoppings, small anisotropy is enough to realize these topological phase transitions. The strained bilayer graphene is considered to have large effective third-nearest-neighbor hoppings ${ }^{14}$.

Therefore, these topological phase transitions can be realized in the ultracold atoms on the optical lattice, strained monolayer graphene or strained bilayer graphene. 


\section{Appendix A: Dirac points in a symmetric system} $\left(C_{6 v}\right)$

We study the system with $C_{6 v}$ symmetry in this Appendix, i.e., we set

$$
t \equiv t_{a}=t_{b}=t_{c}
$$

and

$$
t_{3} \equiv t_{3 a}=t_{3 b}=t_{3 c} .
$$

Then $\epsilon_{1}(\mathbf{k})$ and $\epsilon_{2}(\mathbf{k})$ (Eqs. (10) and (11)) are written as

$$
\begin{aligned}
\epsilon_{1}(\mathbf{k})= & -t\left[\cos \left(\frac{\sqrt{3}}{3} k_{x}\right)+2 \cos \left(\frac{\sqrt{3}}{6} k_{x}\right) \cos \left(\frac{1}{2} k_{y}\right)\right] \\
& -t_{3}\left[\cos \left(\frac{2 \sqrt{3}}{3} k_{x}\right)+2 \cos \left(\frac{\sqrt{3}}{3} k_{x}\right) \cos \left(k_{y}\right)\right],
\end{aligned}
$$

and

$$
\begin{aligned}
\epsilon_{2}(\mathbf{k})= & -t\left[\sin \left(\frac{\sqrt{3}}{3} k_{x}\right)-2 \sin \left(\frac{\sqrt{3}}{6} k_{x}\right) \cos \left(\frac{1}{2} k_{y}\right)\right] \\
& +t_{3}\left[\sin \left(\frac{2 \sqrt{3}}{3} k_{x}\right)-2 \sin \left(\frac{\sqrt{3}}{3} k_{x}\right) \cos \left(k_{y}\right)\right] .
\end{aligned}
$$

In Figures 2, 10, 11, and 12, we plot the lines for $\epsilon_{1}(\mathbf{k})=0$ (thin blue lines) and for $\epsilon_{2}(\mathbf{k})=0$ (thick green lines). The massless Dirac points are given by the intersection points of the blue lines and the green lines. We obtain $\epsilon_{2}(\mathbf{k})=0$ if $k_{x}=0$ from Eq. (A4). From the symmetry of the system, we obtain that $\epsilon_{2}(\mathbf{k})=0$ on the thick green lines in Fig. 2 (a). When $t_{3}=0$ there are no other solutions of $\epsilon_{2}(\mathbf{k})=0$.

In order to find other solutions of $\epsilon_{2}(\mathbf{k})=0$ in the case of $t_{3} \neq 0$, we expand $\epsilon_{2}(\mathbf{k})$ around $k_{x}=0$ and obtain

$$
\begin{aligned}
\epsilon_{2}(\mathbf{k}) & \approx \frac{\sqrt{3}}{3}\left(-t+t \cos \frac{k_{y}}{2}+2 t_{3}-2 t_{3} \cos k_{y}\right) k_{x} \\
& +O\left(k_{x}^{2}\right) .
\end{aligned}
$$

The intersection points of green lines $\left(\epsilon_{2}(\mathbf{k})=0\right)$ and $k_{y}$ axis are obtained by the equation

$$
\frac{\sqrt{3}}{3}\left(-t+t \cos \frac{k_{y}^{(2)}}{2}+2 t_{3}-2 t_{3} \cos k_{y}^{(2)}\right)=0 .
$$

We obtain

$$
\cos \frac{k_{y}^{(2)}}{2}=\frac{t \pm\left|t-8 t_{3}\right|}{8 t_{3}}
$$

This equation has the solution $k_{y}^{(2)}=2 n \pi$ with integers $n$, and other solutions exist if $t_{3}>t / 8$. As seen in Fig. 2,
10,11 , and 12 , there are circular-like green line around $\mathbf{k}=0$. Note that all $\Gamma$ points are not equivalent if we consider $\epsilon_{1}(\mathbf{k})$ and $\epsilon_{2}(\mathbf{k})$ separately, while they are equivalent if we consider $\sqrt{\left(\epsilon_{1}(\mathbf{k})\right)^{2}+\left(\epsilon_{2}(\mathbf{k})\right)^{2}}$.

As seen in Fig. 2(b) and Fig. 10, if $t_{3}<t / 3$, Dirac points are only at $\mathbf{K}$ and $\mathbf{K}^{\prime}$, since green lines and blue lines intersect only at $\mathbf{K}$ and $\mathbf{K}^{\prime}$. In order to examine the number of Dirac points, we also expand $\epsilon_{1}(\mathbf{k})$ with respect to $k_{x}$ around $k_{x}=0$. Then we obtain

$$
\begin{aligned}
\epsilon_{1}(\mathbf{k}) & \approx-t\left(1+2 \cos \frac{k_{y}}{2}\right)-t_{3}\left(1+2 \cos k_{y}\right) \\
& +O\left(k_{x}\right) .
\end{aligned}
$$

We obtain from $\epsilon_{1}(\mathbf{k})=0$ at $k_{x}=0$ that

$$
\cos \frac{k_{y}^{(1)}}{2}=\frac{-t \pm\left|t-2 t_{3}\right|}{4 t_{3}},
$$

which gives the intersection points of blue lines $\left(\epsilon_{1}(\mathbf{k})=\right.$ $0)$ and $k_{y}$ axis. We obtain that the solution of Eq. (A9) is only $k_{y}^{(1)}= \pm 4 \pi / 3+4 n \pi\left(\mathbf{K}\right.$ and $\mathbf{K}^{\prime}$, and their equivalent points) if $t_{3}<t / 3$. If $t_{3}>t / 3$, there exist other solutions of Eq. (A9), which are the intersection points of blue ellipse and $k_{y}$ axis, which we call $\mathbf{D}_{1}$ as shown in Fig. 11 for $t_{3}=0.4 t$. Because of the symmetry the new Dirac points for $t_{3}>t / 3$ make an equilateral triangles around $\mathbf{K}$ and $\mathbf{K}^{\prime}$, as shown in Fig. $11\left(\mathbf{D}_{1}, \mathbf{D}_{2}\right.$ and $\left.\mathbf{D}_{3}\right)$. If $t_{3}=t / 2$, four Dirac points merge at $\mathbf{K}$ and $\mathbf{K}^{\prime}$ as shown in Fig. 12.

\section{Appendix B: Dirac points in an axisymmetric system $\left(C_{2 v}\right)$}

In this appendix we study the system, which has $C_{2 v}$ symmetry, i.e.

$$
\begin{aligned}
t_{b} & =t_{c} \equiv t_{b c}, \\
t_{3 b} & =t_{3 c} \equiv t_{3 b c} .
\end{aligned}
$$

In this case the system has $\pi$ rotational symmetry and the reflectional symmetry with respect to the $x$-axis and the vertical line $x=\sqrt{3} / 6$. We obtain,

$$
\begin{aligned}
\epsilon_{1}(\mathbf{k})= & -t_{a} \cos \left(\frac{\sqrt{3}}{3} k_{x}\right)-2 t_{b c} \cos \left(\frac{\sqrt{3}}{6} k_{x}\right) \cos \left(\frac{1}{2} k_{y}\right) \\
& -t_{3 a} \cos \left(\frac{2 \sqrt{3}}{3} k_{x}\right)-2 t_{3 b c} \cos \left(\frac{\sqrt{3}}{3} k_{x}\right) \cos \left(k_{y}\right),
\end{aligned}
$$

and

$$
\begin{aligned}
\epsilon_{2}(\mathbf{k})= & -t_{a} \sin \left(\frac{\sqrt{3}}{3} k_{x}\right)+2 t_{b c} \sin \left(\frac{\sqrt{3}}{6} k_{x}\right) \cos \left(\frac{1}{2} k_{y}\right) \\
& +t_{3 a} \sin \left(\frac{2 \sqrt{3}}{3} k_{x}\right)-2 t_{3 b c} \sin \left(\frac{\sqrt{3}}{3} k_{x}\right) \cos \left(k_{y}\right) .
\end{aligned}
$$


It is obtained that $\epsilon_{2}(\mathbf{k})=0$ when $k_{x}=0$ and some of the Dirac points are on the $k_{y}$ axis, as in the $C_{6 v}$ case (Appendix A). As in Appendix A, the intersection points of the green lines $\left(\epsilon_{2}(\mathbf{k})=0\right)$ and the $k_{y}$ axis are obtained by

$$
\frac{\sqrt{3}}{3}\left(-t_{a}+t_{b c} \cos \frac{k_{y}^{(2)}}{2}+2 t_{3 a}-2 t_{3 b c} \cos k_{y}^{(2)}\right)=0 .
$$

We obtain

$$
\cos \frac{k_{y}^{(2)}}{2}=\frac{t_{b c} \pm \sqrt{t_{b c}^{2}-16 t_{a} t_{3 b c}+32 t_{3 a} t_{3 b c}+32 t_{3 b c}^{2}}}{8 t_{3 b c}}
$$

The intersection points of the blue lines $\left(\epsilon_{1}(\mathbf{k})=0\right)$ and the $k_{y}$ axis are obtained by

$$
-t_{a}-2 t_{b c} \cos \frac{k_{y}^{(1)}}{2}-t_{3 a}-2 t_{3 b c} \cos k_{y}^{(1)}=0 .
$$

From this equation we obtain

$$
\cos \frac{k_{y}^{(1)}}{2}=\frac{-t_{b c} \pm \sqrt{t_{b c}^{2}-4 t_{a} t_{3 b c}-4 t_{3 a} t_{3 b c}+8 t_{3 b c}^{2}}}{4 t_{3 b c}} .
$$

\section{1. three Dirac points merge}

If the solutions of Eq. (B6) and Eq. (B8) exist and they are the same, three Dirac points $\left(\mathbf{D}_{0}, \mathbf{D}_{2}\right.$ and $\mathbf{D}_{3}$ in Fig. 11) merge at the $k_{y}$ axis as shown in Fig. 21. We obtain that three Dirac points merge on the $k_{y}$ axis when the equation

$$
\left(t_{a}-t_{3 a}-2 t_{3 b c}\right) t_{b c}^{2}+4 t_{3 a}^{2} t_{3 b c}=0
$$

is satisfied. Thus, we obtain that three Dirac points merge on the $k_{y}$ axis if $\left(t_{a}, t_{b}\left(=t_{b c}\right), t_{c}\left(=t_{b c}\right)\right)$ is given by

$$
\left(t_{a}, t_{b}, t_{c}\right)=\left(t_{a}, \frac{2 t_{3 a} \sqrt{t_{3 b c}}}{\sqrt{-t_{a}+t_{3 a}+2 t_{3 b c}}}, \frac{2 t_{3 a} \sqrt{t_{3 b c}}}{\sqrt{-t_{a}+t_{3 a}+2 t_{3 b c}}}\right),
$$

or

$$
\left(t_{a}, t_{b}, t_{c}\right)=\left(t_{3 a}+2 t_{3 b c}-4 \frac{t_{3 a}^{2} t_{3 b c}}{t_{b c}^{2}}, t_{b c}, t_{b c}\right) .
$$

Eq. (B10) is real when $t_{3 a}+2 t_{3 b c}>t_{a}$.

In Fig. 6, Fig. 14 and Fig. 15, we plot the phase diagram in the $t_{b}-t_{c}$ plane by taking $t_{a}=1$ and $t_{3 a}=t_{3 b c} \equiv t_{3}$. We find three Dirac points merge when $t_{b}$ and $t_{c}$ are at the $\mathbf{P}_{1}, \mathbf{P}_{2}$, or $\mathbf{P}_{3}$, which are given in Eqs. (49), (50) and (51). In order to derive $\mathbf{P}_{1}$ we take $t_{a}=1$ in Eq. (B10), and in order to derive $\mathbf{P}_{2}\left(\mathbf{P}_{3}\right)$ we take $t_{b c}=1$ in Eq. (B11) and exchange $t_{a}$ and $t_{c}\left(t_{a}\right.$ and $\left.t_{b}\right)$. For example, if we take $t_{3 a}=t_{3 b c}=4 / 10$, we obtain three Dirac points merge on the $k_{y}$ axis if $\left(t_{a}, t_{b}, t_{c}\right)=$ (a)

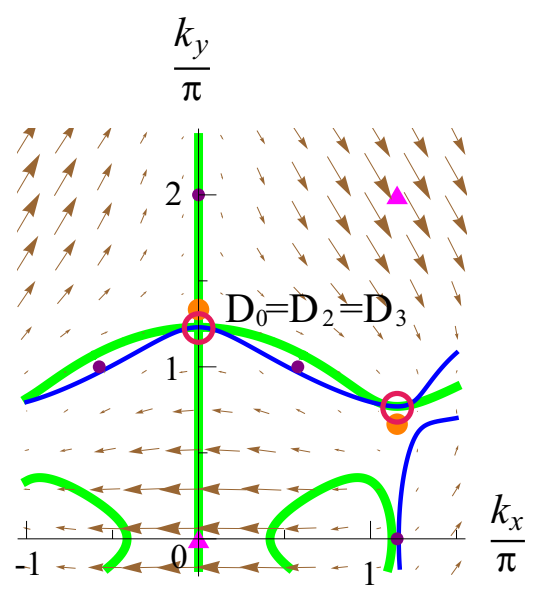

(b)

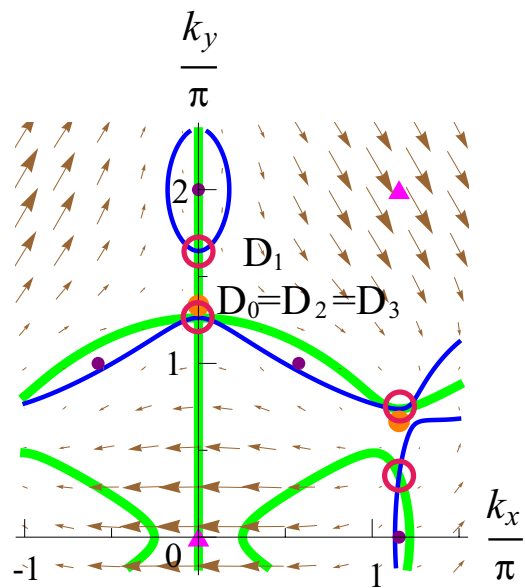

FIG. 21. (Color online) Lines foe $\epsilon_{1}(\mathbf{k})=0$ (thin blue lines) and $\epsilon_{2}(\mathbf{k})=0$ (thick green lines) for (a) $t_{a}=1, t_{b}=t_{c}=$ $1.13137, t_{3 a}=t_{3 b}=t_{3 c}=0.4$, and (b) $t_{a}=0.944, t_{b}=t_{c}=1$, $t_{3 a}=t_{3 b}=t_{3 c}=0.4$. In both cases three Dirac points merge on the $k_{y}$ axis near $\mathbf{K}$ and $\mathbf{K}^{\prime}$. The set of parameters in (a) is $\mathbf{P}_{1}$ in Fig. 15 (b), and the set of parameters in (b) corresponds to $\mathbf{P}_{2}$ and $\mathbf{P}_{3}$ in Fig. 15 (b) with exchanging $t_{a}$ and $t_{b}$. With the anisotropy of $t_{a}$ and $t_{b}=t_{c}$, three Dirac points $\left(\mathbf{D}_{0}, \mathbf{D}_{2}\right.$ and $\left.\mathbf{D}_{3}\right)$ move from those in Fig. 11 and merge.

$(1,4 \sqrt{2} / 5,4 \sqrt{2} / 5) \approx(1,1.13137,1.13137)$, which corresponds to $\mathbf{P}_{1}$ in Fig. $15(\mathrm{~b})$, and $\left(t_{a}, t_{b}, t_{c}\right)=(0.944,1,1)$, which corresponds to $\mathbf{P}_{2}$ and $\mathbf{P}_{3}$ in Fig. 15 (b).

In Fig. 21 we show these two cases. At these parameters three Dirac points, $\mathbf{D}_{0}, \mathbf{D}_{2}$ and $\mathbf{D}_{3}$, merge on the $k_{y}$ axis, as shown in Fig. 21. When parameters are those of Fig. 21 (a), which correspond to $\mathbf{P}_{1}$ in Fig. 15 (b), $\mathbf{D}_{1}$ does not exist, while $\mathbf{D}_{1}$ exists when parameters are those of Fig. 21 (b), which correspond to $\mathbf{P}_{2}$ and $\mathbf{P}_{3}$ in Fig. 15 (b). This difference can be understood by noting the following fact in Fig. 15 (b): When parameters move from the point $t_{a}=t_{b}=t_{c}=1$ to $\mathbf{P}_{1}$, they intersect the 


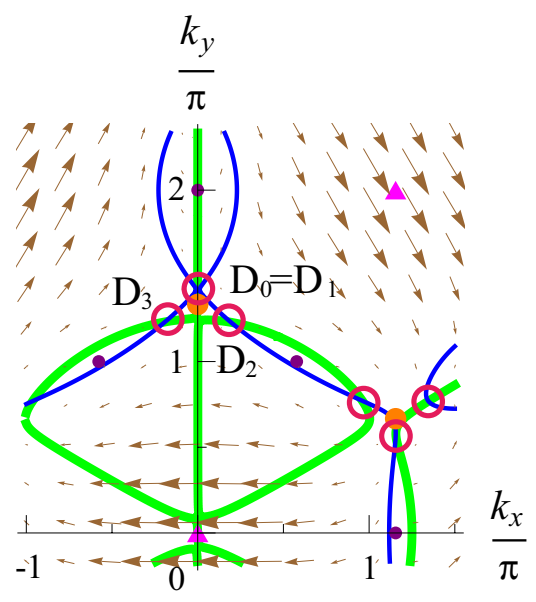

FIG. 22. (Color online) Lines foe $\epsilon_{1}(\mathbf{k})=0$ (thin blue lines) and $\epsilon_{2}(\mathbf{k})=0$ (thick green lines) for $t_{a}=1, t_{b}=t_{c}=0.980$ and $t_{3 a}=t_{3 b}=t_{3 c}=0.4$. This set of parameters is $\mathbf{Q}_{1}$ in Fig. 6 (c).

line $M_{2}$, which shows that Dirac points merge and annihilate at $\mathbf{M}_{2}$ in the Brillouin zone when they cross the line $M_{2}$. On the other hand parameters do not intersect any lines labeled $M_{1}, M_{2}$ and $M_{3}$ when they move from the point $t_{a}=t_{b}=t_{c}=1$ to $\mathbf{P}_{2}$ or $\mathbf{P}_{3}$.

When parameters are changed through these critical values, three Dirac points merges and one Dirac point survives. The topological number (Berry phase) of the Dirac point at $\mathbf{D}_{0}$ is +1 , while these at $\mathbf{D}_{1}, \mathbf{D}_{2}$ and $\mathbf{D}_{3}$ are -1 . As a result, the merged point of three Dirac points has the topological number -1 . However, the merged point is not a simple Dirac point. The energy changes linear in one direction in the wave number and cubic in other direction, and the density of states is proportional to $\epsilon^{1 / 3}$, as we show in Appendix D.

\section{2. two Dirac points merge}

Two Dirac points merge on the $k_{y}$ axis when two solutions of Eq. (B8) become the same, which happens when

$$
t_{b c}^{2}-4 t_{a} t_{3 b c}-4 t_{3 a} t_{3 b c}+8 t_{3 b c}^{2}=0,
$$

and

$$
\left|t_{b c}\right|<4\left|t_{3 b c}\right|
$$

Thus, we obtain that two Dirac points merge on the $k_{y}$ axis if $\left(t_{a}, t_{b}\left(=t_{b c}\right), t_{c}\left(=t_{b c}\right)\right)$ is given by

$$
\left(t_{a}, t_{b}, t_{c}\right)=\left(\frac{t_{b}^{2}}{4 t_{3 b c}}-t_{3 a}+2 t_{3 b c}, t_{b c}, t_{b c}\right),
$$

or

$$
\begin{aligned}
& \left(t_{a}, t_{b}, t_{c}\right) \\
= & \left(t_{a}, 2 \sqrt{\left(t_{a}+t_{3 a}-2 t_{3 b c}\right) t_{3 b c}}, 2 \sqrt{\left(t_{a}+t_{3 a}-2 t_{3 b c}\right) t_{3 b c}}\right) .
\end{aligned}
$$

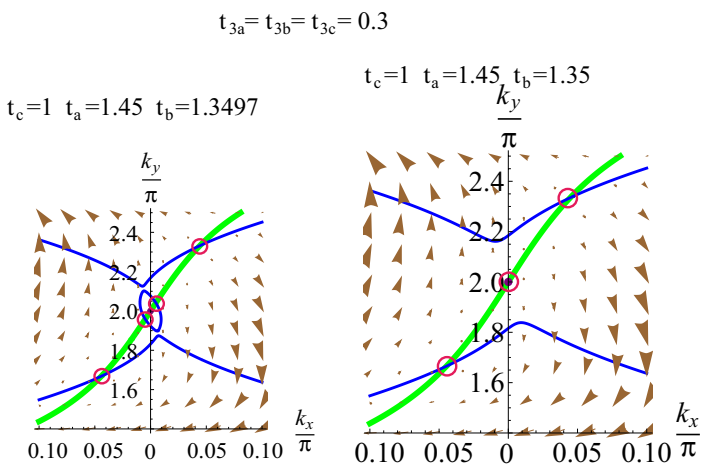

FIG. 23. (Color online) Example for a pair of Dirac points merge at $\mathbf{M}$. Lines of $\epsilon_{1}(\mathbf{k})=0$ (thin blue line) and $\epsilon_{2}(\mathbf{k})=0$ (thick green line).

When $t_{3 a}=t_{3 b c} \equiv t_{3}$ and $t_{a}=1$, we obtain the special points in the phase diagram in $t_{b}-t_{c}$ plane as,

$$
\begin{array}{ll}
\mathbf{Q}_{1}=\left(2 \sqrt{t_{3}\left(1-t_{3}\right)}, 2 \sqrt{t_{3}\left(1-t_{3}\right)}\right) & :\left(t_{3}>1 / 5\right), \\
\mathbf{Q}_{2}=\left(t_{3}+\frac{1}{4 t_{3}}, 1\right) & :\left(t_{3}>1 / 4\right) .
\end{array}
$$

and

$$
\mathbf{Q}_{3}=\left(1, t_{3}+\frac{1}{4 t_{3}}\right) \quad:\left(t_{3}>1 / 4\right) .
$$

For example, if we take $t_{3 a}=t_{3 b c}=4 / 10$, we obtain two Dirac points merge at $\left(t_{a}, t_{b}, t_{c}\right)=$ $(1,2 \sqrt{6} / 5,2 \sqrt{6} / 5) \approx(1,0.980,0.980)$, which corresponds to $\mathbf{Q}_{1}$ in Fig. $15(\mathrm{~b})$, and at $\left(t_{a}, t_{b}, t_{c}\right)=(41 / 40,1,1)=$ $(1.025,1,1)$, which corresponds to $\mathbf{Q}_{2}$ and $\mathbf{Q}_{3}$ in Fig. 15 (b). In these values of parameters, two Dirac points $\mathbf{D}_{0}$ and $\mathbf{D}_{1}$ merge on the $k_{y}$ axis as shown in Fig. 22.

We obtain the phase diagram numerically in Figures 14 and 15. For example, in Fig. 15 (b), the triangularlike region enclosed by the line, $\mathbf{P}_{1}-\mathbf{S}_{1}-\mathbf{Q}_{3}-\mathbf{P}_{2}-\mathbf{Q}_{1}-\mathbf{P}_{3}-\mathbf{Q}_{2}-$ $\mathbf{S}_{2}-\mathbf{P}_{1}$, is the parameter region of $t_{b}$ and $t_{c}$ where the additional Dirac points exist when $t_{a}=1$, and $t_{3 a}=$ $t_{3 b}=t_{3 c}=0.4$. Therefore, the apexes of the triangularlike region in Fig. 15 (b) are $\mathbf{P}_{1}=(1.131,1.131), \mathbf{P}_{2}=$ $(0.994,1)$ and $\mathbf{P}_{3}=(1,0.994)$, and the line passes on $\mathbf{Q}_{1}=(0.980,0.980), \mathbf{Q}_{2}=(1.025,1)$ and $\mathbf{Q}_{3}=(1,1.025)$.

\section{Appendix C: tricritical points}

In this appendix we give the analytical expression for the tricritical points. When $t_{3 a}+2 t_{3 b c}<t_{a}, \mathbf{P}_{1}$ does not exist in the phase phase diagram in the $t_{b} / t_{a}-t_{c} / t_{a}$ plane. In this case the lines $\mathbf{P}_{2}-\mathbf{Q}_{3}$ and $\mathbf{P}_{3}-\mathbf{Q}_{2}$, on which 


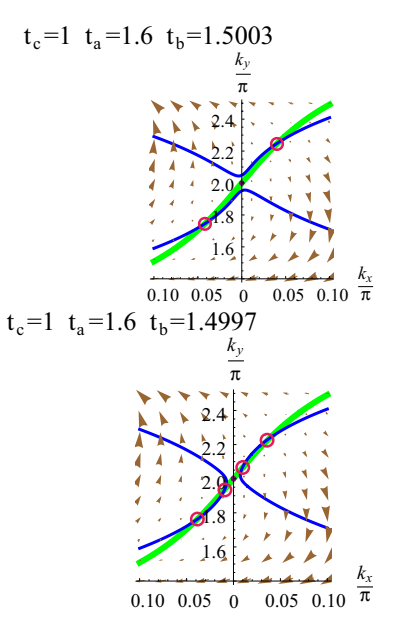

$\mathrm{t}_{3 \mathrm{a}}=\mathrm{t}_{3 \mathrm{~b}}=\mathrm{t}_{3 \mathrm{c}}=0.3$

$$
\mathrm{t}_{\mathrm{c}}=1 \quad \mathrm{t}_{\mathrm{a}}=1.6 \mathrm{t}_{\mathrm{b}}=1.5
$$

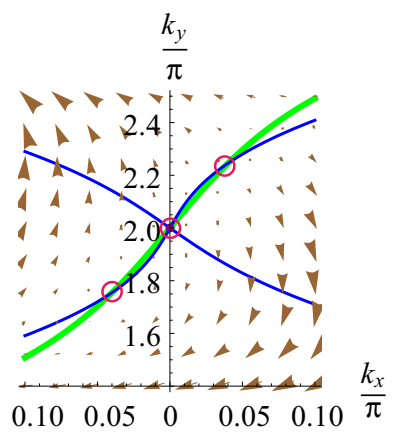

FIG. 24. (Color online) Example for a pair of Dirac points merge at $\mathbf{M}$. Lines of $\epsilon_{1}(\mathbf{k})=0$ (thin blue line) and $\epsilon_{2}(\mathbf{k})=0$ (thick green line).

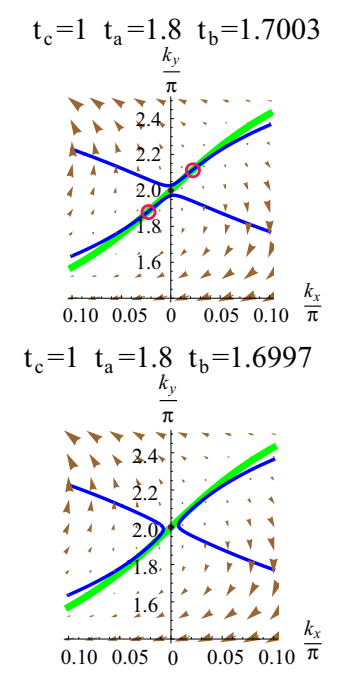

$\mathrm{t}_{3 \mathrm{a}}=\mathrm{t}_{3 \mathrm{~b}}=\mathrm{t}_{3 \mathrm{c}}=0.3$

$\mathrm{t}_{\mathrm{c}}=1 \quad \mathrm{t}_{\mathrm{a}}=1.8 \quad \mathrm{t}_{\mathrm{b}}=1.7$
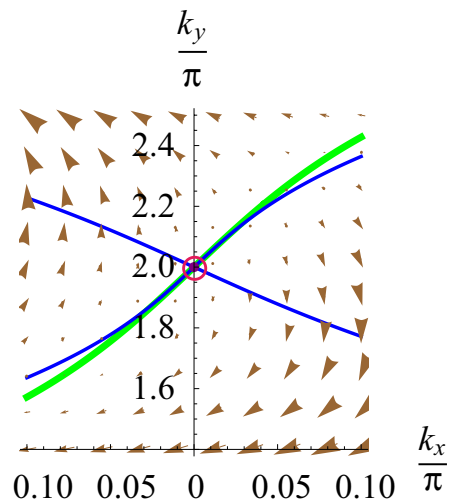

FIG. 25. (Color online) Example for a pair of Dirac points merge at $\mathbf{M}$. Lines of $\epsilon_{1}(\mathbf{k})=0$ (thin blue line) and $\epsilon_{2}(\mathbf{k})=0$ (thick green line).

two Dirac points merge, terminate tangentially on the $M_{1}$ and $M_{3}$ line at the tricritical points, which we call $\mathbf{T}_{3}$ and $\mathbf{T}_{2}$, respectively (see Fig. 14). When $6 t_{3 b c}-t_{3 a}<t_{a}, \mathbf{Q}_{1}$ does not exist, and tricritical points $\left(\mathbf{T}_{1}\right.$ and $\left.\mathbf{T}_{1}^{\prime}\right)$ appear on the $M_{2}$ line. In the following we give the analytical expressions of $\mathbf{T}_{1}, \mathbf{T}_{1}^{\prime}, \mathbf{T}_{2}$ and $\mathbf{T}_{3}$.

We study the case

$$
t_{b}=t_{a}-t_{c}+t_{3 a}+t_{3 b}+t_{3 c} .
$$

Then merged Dirac point (semi-Dirac point) exists at $\mathbf{M}_{2}$ and the equivalent points, for example, $\mathbf{k}=(0,2 \pi)$. We expand $\epsilon_{1}(\mathbf{k})$ and $\epsilon_{2}(\mathbf{k})$ around $\mathbf{k}=(0,2 \pi)$ and obtain

$$
\begin{aligned}
& \epsilon_{1}(\mathbf{k}) \approx \alpha\left(k_{y}-2 \pi\right)^{2}+\beta\left(k_{y}-2 \pi\right) k_{x}+\gamma k_{x}^{2}, \\
& \epsilon_{2}(\mathbf{k}) \approx \delta_{1}\left(k_{y}-2 \pi\right)-\delta_{2} k_{x},
\end{aligned}
$$

where

$$
\begin{aligned}
\alpha & =\frac{1}{8}\left(-t_{a}-t_{3 a}+3 t_{3 b}+3 t_{3 c}\right), \\
\beta & =\frac{\sqrt{3}}{12}\left(t_{a}-2 t_{c}+t_{3 a}-3 t_{3 b}+5 t_{3 c}\right), \\
\gamma & =\frac{1}{8}\left(t_{a}+5 t_{3 a}+t_{3 b}+t_{3 c}\right), \\
\delta_{1} & =\frac{1}{2}\left(t_{a}-2 t_{c}+t_{3 a}+3 t_{3 b}-t_{3 c}\right),
\end{aligned}
$$

and

$$
\delta_{2}=\frac{\sqrt{3}}{2}\left(t_{a}-t_{3 a}+t_{3 b}+t_{3 c}\right) .
$$

The line given by $\epsilon_{2}(\mathbf{k})=0$ goes through $(0,2 \pi)$ and it is obtained as

$$
k_{y}-2 \pi \approx \frac{\delta_{2}}{\delta_{1}} k_{x}
$$

On the other hand there are no lines near $(0,2 \pi)$ given by $\epsilon_{1}(\mathbf{k})=0$, if $\beta^{2}-4 \alpha \gamma<0$. We show the example in Fig. $23\left(t_{a}=1.45, t_{b}=1.35, t_{c}=1\right.$, and $t_{3 a}=t_{3 b}=$ $\left.t_{3 c}=0.3\right)$. If $t_{b}$ is changed smaller, the semi-Dirac point becomes a a pair of Dirac points near $\mathbf{k}=(0,2 \pi)$ as shown in the left figure in Fig. 23.

If $\beta^{2}-4 \alpha \gamma>0, \epsilon_{1}(\mathbf{k})=0$ gives two lines,

$$
k_{y}-2 \pi \approx \frac{-\beta \pm \sqrt{\beta^{2}-4 \alpha \gamma}}{2 \alpha} k_{x}
$$

as shown in Figs. 24 and Figs. $25\left(\left(t_{a}, t_{b}, t_{c}\right)=(1.6,1.5,1)\right.$ and $(1.8,1.7,1)$, respectively, and $\left.t_{3 a}=t_{3 b}=t_{3 c}=0.3\right)$.

As seen in the left figures in Fig. 24, the merged Dirac points at $\mathbf{k}=(0,2 \pi)$ disappear or become two Dirac points, when we change $t_{b}$ larger or smaller, respectively. Since there are other Dirac points in Fig. 24, this topological phase transition separate the phases with two Dirac points and four Dirac points. On the other hand, as seen in the left figures in Fig. 25, the merged Dirac points at $\mathbf{k}=(0,2 \pi)$ become two Dirac points or disappear, when we change $t_{b}$ larger or smaller, respectively. Since there are no other Dirac points in Fig. 25, the topological phase transition is the transition between two phases with two Dirac points and zero Dirac points in this parameters. The tricritical point, on which three phases with zero, two and four Dirac points terminate in the parameter space, happens when one of the lines $\epsilon_{1}(\mathbf{k})=0$ coincides with the line $\epsilon_{2}(\mathbf{k})=0$,

$$
\frac{\delta_{2}}{\delta_{1}}=\frac{-\beta-\sqrt{\beta^{2}-4 \alpha \gamma}}{2 \alpha}
$$


Tricritical points $\mathbf{T}_{1}$ and $\mathbf{T}_{1}^{\prime}$ can be obtained by solving Eq. (C11) with respect to $t_{c}$ as a function of $t_{a}, t_{3 a}, t_{3 b}$ and $t_{3 c}$. We obtain

$$
\begin{aligned}
\tilde{t}_{c \pm} & =\frac{t_{a}+t_{3 a}+t_{3 b}+t_{3 c}}{2}+\frac{2\left(t_{3 b}-t_{3 c}\right) t_{3 a}}{t_{a}+t_{3 a}+t_{3 b}+t_{3 c}} \\
& \pm \frac{t_{a}-t_{3 a}+t_{3 b}+t_{3 c}}{2} \\
& \times \sqrt{\frac{t_{a}+t_{3 a}-3\left(t_{3 b}+t_{3 c}\right)}{t_{a}+t_{3 a}+t_{3 b}+t_{3 c}}+\left(\frac{2\left(t_{3 b}-t_{3 c}\right)}{t_{a}+t_{3 a}+t_{3 b}+t_{3 c}}\right)^{2}} .
\end{aligned}
$$

Since $\mathbf{T}_{1}$ and $\mathbf{T}_{1}^{\prime}$ are on the line $M_{2}$ in the phase diagram in $t_{b}-t_{c}$ plane with $t_{a}=1$, we obtain from Eq. (C12) and taking $t_{a}=1$

$$
\begin{aligned}
\left(\mathbf{T}_{1}\right)_{x} & =\left.\left(1-\tilde{t}_{c-}+t_{3 a}+t_{3 b}+t_{3 c}\right)\right|_{t_{a}=1} \\
& =\frac{1+t_{3 a}+t_{3 b}+t_{3 c}}{2}-\frac{2\left(t_{3 b}-t_{3 c}\right) t_{3 a}}{1+t_{3 a}+t_{3 b}+t_{3 c}} \\
& +\frac{1-t_{3 a}+t_{3 b}+t_{3 c}}{2} \\
& \times \sqrt{\frac{1+t_{3 a}-3\left(t_{3 b}+t_{3 c}\right)}{1+t_{3 a}+t_{3 b}+t_{3 c}}+\left(\frac{2\left(t_{3 b}-t_{3 c}\right)}{1+t_{3 a}+t_{3 b}+t_{3 c}}\right)^{2}},
\end{aligned}
$$

$$
\begin{aligned}
\left(\mathbf{T}_{1}\right)_{y} & =\left.\left(\tilde{t}_{c-}\right)\right|_{t_{a}=1} \\
& =\frac{1+t_{3 a}+t_{3 b}+t_{3 c}}{2}+\frac{2\left(t_{3 b}-t_{3 c}\right) t_{3 a}}{1+t_{3 a}+t_{3 b}+t_{3 c}} \\
& -\frac{1-t_{3 a}+t_{3 b}+t_{3 c}}{2} \\
& \times \sqrt{\frac{1+t_{3 a}-3\left(t_{3 b}+t_{3 c}\right)}{1+t_{3 a}+t_{3 b}+t_{3 c}}+\left(\frac{2\left(t_{3 b}-t_{3 c}\right)}{1+t_{3 a}+t_{3 b}+t_{3 c}}\right)^{2}},
\end{aligned}
$$

$$
\begin{aligned}
\left(\mathbf{T}_{1}^{\prime}\right)_{x} & =\left.\left(1-\tilde{t}_{c+}+t_{3 a}+t_{3 b}+t_{3 c}\right)\right|_{t_{a}=1} \\
& =\frac{1+t_{3 a}+t_{3 b}+t_{3 c}}{2}-\frac{2\left(t_{3 b}-t_{3 c}\right) t_{3 a}}{1+t_{3 a}+t_{3 b}+t_{3 c}} \\
& -\frac{1-t_{3 a}+t_{3 b}+t_{3 c}}{2} \\
& \times \sqrt{\frac{1+t_{3 a}-3\left(t_{3 b}+t_{3 c}\right)}{1+t_{3 a}+t_{3 b}+t_{3 c}}+\left(\frac{2\left(t_{3 b}-t_{3 c}\right)}{1+t_{3 a}+t_{3 b}+t_{3 c}}\right)^{2}},
\end{aligned}
$$

and

$$
\begin{aligned}
\left(\mathbf{T}_{1}^{\prime}\right)_{y} & =\left.\left(\tilde{t}_{c+}\right)\right|_{t_{a}=1} \\
& =\frac{t_{a}+t_{3 a}+t_{3 b}+t_{3 c}}{2}+\frac{2\left(t_{3 b}-t_{3 c}\right) t_{3 a}}{t_{a}+t_{3 a}+t_{3 b}+t_{3 c}} \\
& +\frac{t_{a}-t_{3 a}+t_{3 b}+t_{3 c}}{2} \\
& \times \sqrt{\frac{t_{a}+t_{3 a}-3\left(t_{3 b}+t_{3 c}\right)}{t_{a}+t_{3 a}+t_{3 b}+t_{3 c}}+\left(\frac{2\left(t_{3 b}-t_{3 c}\right)}{t_{a}+t_{3 a}+t_{3 b}+t_{3 c}}\right)^{2}} .
\end{aligned}
$$

We obtain Eqs. (52) and (53) by taking $t_{3 a}=t_{3 b}=t_{3 c}=$ $t_{3}$ in Eqs. (C13), (C14), (C15) and (C16).

The tricritical points $\mathbf{T}_{2}$ and $\mathbf{T}_{3}$ are obtained as follows. By solving Eq. (C11) with respect to $t_{a}$, we obtain the critical value of $t_{a}$ as a function of $t_{c}, t_{3 a}, t_{3 b}$ and $t_{3 c}$ as

$$
\begin{aligned}
\tilde{t}_{a} & =\frac{t_{c}-t_{3 a}-t_{3 b}-t_{3 c}}{2}+\frac{2\left(t_{3 a}-t_{3 b}\right) t_{3 c}}{t_{c}-t_{3 a}-t_{3 b}-t_{3 c}} \\
& +\frac{t_{c}-t_{3 a}-t_{3 b}+t_{3 c}}{2} \\
& \times \sqrt{\frac{t_{c}+3 t_{3 a}+3 t_{3 b}-t_{3 c}}{t_{c}-t_{3 a}-t_{3 b}-t_{3 c}}+\left(\frac{2\left(t_{3 a}-t_{3 b}\right)}{t_{c}-t_{3 a}-t_{3 b}-t_{3 c}}\right)^{2}} .
\end{aligned}
$$

The tricritical point $\mathbf{T}_{2}$ is on the line $M_{3}$ in the phase diagram in $t_{b}-t_{c}$ plane, i.e., the merged Dirac points are located at $\mathbf{M}_{3}$ instead of $\mathbf{M}_{2}$. Since the above calculation has been done for $\mathbf{M}_{2}$, we change $t_{a} \rightarrow t_{b} \rightarrow t_{c} \rightarrow t_{a}$ and $t_{3 a} \rightarrow t_{3 b} \rightarrow t_{3 c} \rightarrow t_{3 a}$, cyclically, in order to obtain $\mathbf{T}_{2}$. Then taking $t_{a}=1$, we obtain

$$
\begin{aligned}
\left(\mathbf{T}_{2}\right)_{x} & =\frac{1-t_{3 a}-t_{3 b}-t_{3 c}}{2}+\frac{2\left(t_{3 b}-t_{3 c}\right) t_{3 a}}{1-t_{3 a}-t_{3 b}-t_{3 c}} \\
& +\frac{1+t_{3 a}-t_{3 b}-t_{3 c}}{2} \\
& \times \sqrt{\frac{1-t_{3 a}+3 t_{3 b}+3 t_{3 c}}{1-t_{3 a}-t_{3 b}-t_{3 c}}+\left(\frac{2\left(t_{3 b}-t_{3 c}\right)}{1-t_{3 a}-t_{3 b}-t_{3 c}}\right)^{2}},
\end{aligned}
$$

and

$$
\begin{aligned}
\left(\mathbf{T}_{2}\right)_{y} & =\left(\mathbf{T}_{2}\right)_{x}-1+t_{3 a}+t_{3 b}+t_{3 c} \\
& =-\frac{1-t_{3 a}-t_{3 b}-t_{3 c}}{2}+\frac{2\left(t_{3 b}-t_{3 c}\right) t_{3 a}}{1-t_{3 a}-t_{3 b}-t_{3 c}} \\
& +\frac{1+t_{3 a}-t_{3 b}-t_{3 c}}{2} \\
& \times \sqrt{\frac{1-t_{3 a}+3 t_{3 b}+3 t_{3 c}}{1-t_{3 a}-t_{3 b}-t_{3 c}}+\left(\frac{2\left(t_{3 b}-t_{3 c}\right)}{1-t_{3 a}-t_{3 b}-t_{3 c}}\right)^{2}} .
\end{aligned}
$$

We obtain Eq. (54) by taking $t_{3 a}=t_{3 b}=t_{3 c}=t_{3}$ in Eqs. (C18) and (C19).

We can obtain $\mathbf{T}_{3}$ by changing $t_{3 a} \rightarrow t_{3 b} \rightarrow t_{3 c} \rightarrow t_{3 a}$, cyclically, in Eqs. (54) and (54). Then we obtain Eq. (55) similarly.

\section{Appendix D: Density of states}

We study the density of states $D(\epsilon)$ for $|\epsilon| \ll 1$ in this Appendix.

\section{Density of states due to Dirac points}

First we study the Density of states due to Dirac point. A Dirac point $\left(\mathbf{k}^{*}\right)$ is given by the intersection points 
of two lines, $\epsilon_{1}(\mathbf{k})=0$ and $\epsilon_{2}(\mathbf{k})=0$, in the Brillouin zone. Two lines can be approximated as straight lines near the Dirac points. We take power series expansions of $\epsilon_{1}(\mathbf{k})$ and $\epsilon_{2}(\mathbf{k})$ about $\mathbf{k}^{*}$. By rotating the $k_{x}-k_{y}$ axes appropriately and taking $\mathbf{k}^{*}=0$ for simplicity, we can write

$$
\begin{aligned}
& \epsilon_{1}(\mathbf{k}) \approx \frac{1}{\sqrt{2}} C\left(k_{x}+u k_{y}\right), \\
& \epsilon_{2}(\mathbf{k}) \approx \frac{1}{\sqrt{2}} C^{\prime}\left(k_{x}-u k_{y}\right),
\end{aligned}
$$

where $C(>0), C^{\prime}(>0)$ and $u(>0)$ are constants. The density of states due to this Dirac point is calculated as

$$
\begin{aligned}
D(\epsilon) & =\frac{1}{S_{B Z}} \iint d k_{x} d k_{y} \delta\left(|\epsilon|-\sqrt{\left(\epsilon_{1}(\mathbf{k})\right)^{2}+\left(\epsilon_{2}(\mathbf{k})\right)^{2}}\right) \\
& =\frac{1}{S_{B Z}} \frac{\pi}{C C^{\prime} u}|\epsilon|,
\end{aligned}
$$

where $S_{B Z}$ is the area of the Brillouin zone. We obtain that the density of states is proportional to $|\epsilon|$.

\section{Density of states due to merged Dirac points}

The merged Dirac points at $\mathbf{k}=\mathbf{k}^{*}$ are classified into four types;

(a) Two lines which are given by $\epsilon_{1}(\mathbf{k})=0$ and $\epsilon_{2}(\mathbf{k})=$ 0 touch in the order $n$ at $\mathbf{k}=\mathbf{k}^{*}$.

(b) One of the lines makes a loop and it shrinks into a point at $\mathbf{k}=\mathbf{k}^{*}$.

(c) The equation $\epsilon_{2}(\mathbf{k})=0$ gives two intersecting lines at $\mathbf{k}=\mathbf{k}^{*}$ and the line given by the equation $\epsilon_{1}(\mathbf{k})=0$ touches with one of the line in the order $m$ at $\mathbf{k}=\mathbf{k}^{*}$.

(d) Both equations, $\epsilon_{1}(\mathbf{k})=0$ and $\epsilon_{2}(\mathbf{k})=0$ give two lines intersecting at $\mathbf{k}=\mathbf{k}^{*}$.

There may be other possibilities, for example more than two lines are given by the equation $\epsilon_{2}(\mathbf{k})=0$ in type (c) and (d), or two lines given by $\epsilon_{2}(\mathbf{k})=0$ touch each other with order $\ell(\geq 2)$ at $\mathbf{k}=\mathbf{k}^{*}$ in type (c). We neglect these possibilities for simplicity, since these cases are not realized in our present study.

When $t_{a}=t_{b}=t_{c}=1$ and $t_{3 a}=t_{3 b}=t_{3 c}=1 / 3$, two Dirac points merge at $\mathbf{M}_{1}, \mathbf{M}_{2}$ and $\mathbf{M}_{3}$ (see Fig. 10). At $\mathbf{k}=(2 \sqrt{3} \pi / 3,0)$, two lines touch quadratically $(n=2)$, which is type (a). At $\mathbf{k}=(0, \pi)$, which is the equivalent point to $\mathbf{k}=(2 \sqrt{3} \pi / 3,0)$, the line given by the equation $\epsilon_{1}(\mathbf{k})=0$ shrinks into a point (compare with Fig. 11), which is type (b). Although the merged Dirac point at $\mathbf{k}=(0, \pi)$ looks different from that at $\mathbf{k}=(2 \sqrt{3} \pi / 3,0)$, the energy around these points $\sqrt{\left(\epsilon_{1}(\mathbf{k})\right)^{2}+\left(\epsilon_{2}(\mathbf{k})\right)^{2}}$ is the same. Type (c) of the merged Dirac points can be seen in Fig. 22, where two Dirac points, $\mathbf{D}_{0}$ and $\mathbf{D}_{1}$, merge on the $k_{y}$ axis when $t_{a}=1, t_{b}=t_{c}=0.980$, and $t_{3 a}=t_{3 b}=t_{3 c}=0.4$.

When the parameters are at the tricritical points in the phase diagram $\left(\mathbf{T}_{1}, \mathbf{T}_{1}^{\prime}, \mathbf{T}_{2}\right.$ and $\left.\mathbf{T}_{3}\right), \epsilon_{1}(\mathbf{k})=0$ gives two lines and one of the lines touches cubically with the line given by $\epsilon_{2}(\mathbf{k})=0$ (see the point $\mathbf{k}=(0,2 \pi)$ in Fig. 24 and Fig. 25). This is type (c) with cubic touching $(m=3)$. Four Dirac points with the topological numbers $+1,+1,-1$ and -1 merge at this point. In the same parameters for the tricritical point, type (a) with $n=4$ is realized at $\mathbf{k}=(2 \sqrt{3} \pi / 3,0)$, (not shown).

First we study the density states for the merged Dirac points of type (a). If two lines touch in the order $n$, we perform the affine transformation, i.e. rotation, translation, and stretching in the momentum space, and we can write

$$
\begin{aligned}
\epsilon_{1}^{(a)}(\mathbf{k}) & \approx \frac{1}{\sqrt{2}}\left(k_{x}+u_{n} k_{y}^{n}\right), \\
\epsilon_{2}^{(a)}(\mathbf{k}) & \approx \frac{1}{\sqrt{2}}\left(k_{x}-u_{n} k_{y}^{n}\right),
\end{aligned}
$$

where $u_{n}(>0)$ is a constant. The topological number at this point is \pm 1 if $n$ is odd and 0 if $n$ is even. In this case we obtain

$$
\sqrt{\left(\epsilon_{1}^{(a)}(\mathbf{k})\right)^{2}+\left(\epsilon_{2}^{(a)}(\mathbf{k})\right)^{2}}=\sqrt{k_{x}^{2}+u_{n}^{2} k_{y}^{2 n}} .
$$

The density of states is obtained as

$$
D^{(a)}(\epsilon)=\frac{1}{S_{B Z}^{\prime}} \frac{2 \sqrt{\pi} \Gamma\left(\frac{2 n+1}{2 n}\right)}{\left(u_{n}\right)^{\frac{1}{n}} \Gamma\left(\frac{n+1}{2 n}\right)}|\epsilon|^{\frac{1}{n}},
$$

where $S_{B Z}^{\prime}$ is the area of the Brillouin zone after the affine transformation. We obtain the Dirac point by taking $n=1$. When two Dirac points merge, we obtain a semi-Dirac point (energy depend linearly on the one direction of the momentum and quadratically in the other direction), which is obtained by taking $n=2$. If three Dirac points merge, $n=3$ is realized, as seen in the merged points near $\mathbf{k}=(2 \sqrt{3} \pi / 3,2 \pi / 3)$ in Fig. 21. At the tricritical point in the phase diagram in the parameter space, $n=4$ is realized (at $\mathbf{k}=(2 \sqrt{3} \pi / 3,0)$ for the parameters in Fig. 24 and Fig. 25, although that point is not shown in these figures.

Next we study the density of states due to the merged Dirac point of type (b). Type (b) of the merged Dirac points can be described after the affine transformation as

$$
\begin{aligned}
& \epsilon_{1}^{(b)}(\mathbf{k}) \approx k_{x}, \\
& \epsilon_{2}^{(b)}(\mathbf{k}) \approx C^{\prime \prime}\left(k_{x}^{2}+k_{y}^{2}\right),
\end{aligned}
$$

where $C^{\prime \prime}(>0)$ is a constant. In this simplification $\epsilon_{1}^{(b)}(\mathbf{k})=0$ gives a straight line and $\epsilon_{2}^{(b)}(\mathbf{k})=0$ gives the point $\mathbf{k}=(0,0)$. We obtain $\sqrt{\left(\epsilon_{1}^{(b)}(\mathbf{k})\right)^{2}+\left(\epsilon_{2}^{(b)}(\mathbf{k})\right)^{2}}=$ $\left|k_{x}\right|+O\left(k_{x}^{2}\right)$ in the $k_{x}$ direction and $C^{\prime \prime} k_{y}^{2}$ in the $k_{y}$ direction. Therefore, Eqs. (D8) and (D9) describe semi-Dirac 
points and the density of states is proportional to $\sqrt{\epsilon}$. Indeed, the density of states is calculated as

$$
\begin{aligned}
D^{(b)}(\epsilon) & \propto \int \frac{2|\epsilon|}{\sqrt{C^{\prime \prime}} \sqrt{\epsilon^{2}-k_{x}^{2}} \sqrt{\sqrt{\epsilon^{2}-k_{x}^{2}}-C^{\prime \prime} k_{x}^{2}}} d k_{x} \\
& =\frac{2 \sqrt{|\epsilon|}}{\sqrt{C^{\prime \prime}}} \int \frac{1}{\sqrt{1-x^{2}} \sqrt{\sqrt{1-x^{2}}-C^{\prime \prime} \epsilon^{2} x^{2}}} d x \\
& \approx \frac{2 \sqrt{\pi} \Gamma\left(\frac{1}{4}\right)}{\sqrt{C^{\prime \prime}} \Gamma\left(\frac{3}{4}\right)} \sqrt{|\epsilon|},
\end{aligned}
$$

where integral should be performed where the integrand is real and we have neglected higher order terms in $\epsilon$.
The merged Dirac point of type (c) is described by

$$
\begin{aligned}
& \epsilon_{1}^{(c)}(\mathbf{k}) \approx k_{x}, \\
& \epsilon_{2}^{(c)}(\mathbf{k}) \approx k_{y}\left(k_{x}-u^{\prime} k_{y}^{m}\right) .
\end{aligned}
$$

In this case $\epsilon_{1}^{(c)}(\mathbf{k})=0$ gives a straight line and $\epsilon_{2}^{(c)}(\mathbf{k})=$ 0 gives an intersecting straight line and a curve touching to the first line at $\mathbf{k}=(0,0)$ in the $m$ th order (if $m=$ $1, \epsilon_{2}^{(c)}(\mathbf{k})=0$ gives two intersecting lines). We obtain $\sqrt{\left(\epsilon_{1}^{(c)}(\mathbf{k})\right)^{2}+\left(\epsilon_{2}^{(c)}(\mathbf{k})\right)^{2}}=\left|k_{x}\right|$ in the $k_{x}$ direction and $u^{\prime}\left|k_{y}\right|^{m+1}$ in the $k_{y}$ direction. Therefore, type (c) with $m$ is similar to type (a) with $n=m+1$. We obtain the density of states due to this merged points as

$$
D(\epsilon)^{(c)} \propto|\epsilon|^{\frac{1}{m+1}} .
$$

The simplest case of the type (d) is realized in the case $t_{a}=t_{b}=t_{c}=1$ and $t_{3 a}=t_{3 b}=t_{3 c}=1 / 2$ as discussed in Section VIA. In that case the topological number is two and the density of states is independent of energy for $|\epsilon| \approx 0$.
${ }^{1}$ K. S. Novoselov, A. K. Geim, S. V. Morozov, D. Jiang, Y. Zhang, S. V. Dubonos, I. V. Grigorieva, and A. A. Firsov, Science 306, 666 (2004).

2 K. S. Novoselov, A. K. Geim, S. V. Morozov, D. Jiang, M. I. Katsnelson, I. V. Grigorieva, S. V. Dubonos, and A. A. Firsov, Nature 438, 197 (2005).

3 Y. Zhang, Y.-W. Tan, H. L. Stormer, and P. Kim, Nature 438, 201 (2005).

4 S. Katayama, A. Kobayashi, and Y. Suzumura, J. Phys. Soc. of Jpn. 75, 023708 (2006).

${ }^{5}$ N. Tajima, S. Sugawara, R. Kato, Y. Nishio, and K. Kajita, Phys. Rev. Lett. 102, 176403 (2009).

6 P. Richard, K. Nakayama, T. Sato, M. Neupane, Y.-M. Xu, J. H. Bowen, G. F. Chen, J. L. Luo, N. L. Wang, X. Dai, Z. Fang, H. Ding, and T. Takahashi, Phys. Rev. Lett. 104, 137001 (2010).

7 Y. Hasegawa, R. Konno, H. Nakano, and M. Kohmoto, Phys. Rev. B 74, 033413 (2006).

8 L. Tarruell, D. Greif, T. Uehlinger, G. Jotzu, and T. Esslinger, Nature 483, 302 (2012).

9 G. Montambaux, F. Piéchon, J.-N. Fuchs, and M. O. Goerbig, Phys. Rev. B 80, 153412 (2009).

10 Y.-W. Son, S.-M. Choi, Y. P. Hong, S. Woo, and S.-H. Jhi, Phys. Rev. B 84, 155410 (2011).

11 M. Mucha-Kruczyński, I. L. Aleiner, and V. I. Fal'ko, Phys. Rev. B 84, 041404 (2011).

12 R. de Gail, M. O. Goerbig, F. Guinea, G. Montambaux, and A. H. Castro Neto, Phys. Rev. B 84, 045436 (2011).

13 R. de Gail, M. O. Goerbig, and G. Montambaux, Phys. Rev. B 86, 045407 (2012).
14 G. Montambaux, arXiv:1205.4459v1 [cond-mat.mes-hall] (2012).

15 C. Bena and L. Simon, Phys. Rev. B 83, 115404 (2011).

16 M. Farjam and H. Rafii-Tabar, Phys. Rev. B 80, 167401 (2009).

17 G. Gui, J. Li, and J. Zhong, Phys. Rev. B 80, 167402 (2009).

18 S.-M. Choi, S.-H. Jhi, and Y.-W. Son, Phys. Rev. B 81, 081407 (2010).

19 P. R. Wallace, Phys. Rev. 71, 622 (1947).

20 S. Reich, J. Maultzsch, C. Thomsen, and P. Ordejón, Phys. Rev. B 66, 035412 (2002).

${ }^{21}$ K. Kishigi, H. Hanada, and Y. Hasegawa, J. Phys. Soc. Jpn. 77, 074707 (2008).

${ }^{22}$ K. Kishigi, R. Takeda, and Y. Hasegawa, Journal of Physics: Conference Series 132, 012005 (2008).

23 S. Y. Zou, G.-H. Gweon, A. V. Fedorov, P. N. First, W. A. de Heer, D.-H. Lee, F. Guinea, A. H. C. Neto, and A. Lanzara, Nat. Phys. 6, 770 (2007).

24 V. M. Pereira, A. H. C. Neto, and N. M. R. Peres, Phys. Rev. B 80, 045401 (2009).

25 G. Cocco, E. Cadelano, and L. Colombo, Phys. Rev. B 81, 241412 (2010).

${ }^{26}$ K. Kishigi, K. Ueno, E. Miyamoto, and Y. Hasegawa, Journal of Physics: Conference Series 334, 012047 (2011).

27 S. Banerjee, R. R. P. Singh, V. Pardo, and W. E. Pickett, Phys. Rev. Lett. 103, 016402 (2009).

28 P. Dietl, F. Piéchon, and G. Montambaux, Phys. Rev. Lett. 100, 236405 (2008). 